\title{
EL GRADO DE CONOCIMIENTO Y UTILIZACIÓN DEL CUADRO DE MANDO INTEGRAL EN LOS AYUNTAMIENTOS PORTUGUESES
}

\section{THE LEVEL OF KNOWLEDGE AND USE OF THE BALANCED SCORECARD IN THE PORTUGUESE CITY COUNCILS}

\author{
Patrícia Rodrigues Quesado ${ }^{1}$; Beatriz Aibar Guzmán² : Lúcia Lima Rodrigues $^{3}$ \\ ${ }^{1}$ Instituto Politécnico do Cávado e do Ave - IPCA - Barcelos - Portugal \\ pquesado@ipca.pt \\ ${ }^{2}$ Universidade de Santiago de Compostela - USC - Santiago de Compostela - Espanha \\ beatriz.aibar@usc.es \\ ${ }^{3}$ Universidade do Minho - UM - Braga - Portugal \\ lrodrigues@eeg.uminho.pt
}

\begin{abstract}
Resumen
La búsqueda de la racionalización en la gestión de los recursos públicos y la necesidad de mejorar la calidad de los servicios prestados, aspectos asociados al modelo de "Nueva Gestión Pública", han motivado la aplicación en las entidades públicas de conceptos y prácticas desarrollados y utilizados previamente en el ámbito privado. En este sentido, varias entidades públicas y sin ánimo de lucro han manifestado particular interés en la implementación del Cuadro de Mando Integral (CMI). Este trabajo tiene como objetivo proporcionar algunas ideas acerca de la importancia, las características y las necesarias adaptaciones del CMI para su aplicación a organizaciones públicas y sin ánimo de lucro, así como averiguar si los ayuntamientos portugueses conocen y están a adoptar el CMI. En consecuencia, hemos enviado un cuestionario a los 308 ayuntamientos portugueses, con una tasa de respuesta del 32,5\%. Los resultados obtenidos permiten concluir que aunque la mayoría de los encuestados conoce el CMI, su utilización en dichas organizaciones es muy reducida.
\end{abstract}

Palabras-clave: cuadro de mando integral; sector público; contabilidad de gestión.

\section{Introducción}

La consideración de los ciudadanos como "clientes" de los bienes y servicios proporcionados por las administraciones públicas y la adopción por parte de éstas de una mayor orientación a los resultados son algunas de las razones que han llevado a las entidades públicas y sin ánimo de lucro a desarrollar e implantar un conjunto de indicadores de gestión que permitan la mejor asignación de los recursos disponibles. Sin embargo, la constatación, por un lado, de que la utilización de dichos indicadores de forma aislada e individualizada impide obtener una imagen 
global de la actuación y el rendimiento de la organización y, por otro, de la sobrecarga informativa que supone el contar con una batería de medidas inconexas e independientes hizo necesario desarrollar modelos que fuesen capaces de integrar los distintos indicadores y, al mismo tiempo, vincularlos a los objetivos y la estrategia de la entidad. En este sentido, el Cuadro de Mando Integral (CMI) ha sido considerado como una herramienta de gestión global y estratégica útil para la modernización del sector público, puesto que permite vertebrar las diversas medidas o indicadores relativos a las diferentes áreas de actuación, de tal forma que ofrezca un marco de referencia completo en base al cual evaluar la gestión desarrollada. Dado que el CMI ofrece una visión global e integrada de la entidad y que los indicadores desarrollados están vinculados a los objetivos y metas establecidos (y, por consiguiente, a la estrategia de la organización), su utilización como instrumento de gestión evita que se produzcan vacíos a nivel de ejecución y consistencia de estrategias (WILSON et al., 2003; BASTIDAS y RIPOLL, 2003; HORVÁTH \& PARTNERS, 2003).

El presente artículo tiene como objetivo proporcionar algunas ideas acerca de la importancia, las características y las necesarias adaptaciones del CMI para su aplicación a organizaciones públicas y sin ánimo de lucro, así como averiguar si los ayuntamientos portugueses conocen y están a adoptar el CMI. De acuerdo con este objetivo, hemos estructurado el trabajo de la siguiente forma: inicialmente se exponen brevemente algunos aspectos teóricos relativos a las características del CMI en el sector público, analizando las reestructuraciones a realizar a nivel de las perspectivas propuestas inicialmente por Kaplan y Norton (1992, 1996a, 1996b, 2000, 2001). Con esta base, se presentan modelos basados en el CMI específicamente desarrollados para el sector público y se analiza su proceso de implantación. A continuación, presentamos las principales ventajas y aspectos críticos resultantes de su adopción, así como diversos estudios realizados en distintos países sobre esta temática. Finalmente, presentamos un estudio realizado en ayuntamientos portugueses con el objetivo de obtener evidencia empírica sobre el conocimiento y utilización del CMI en dichas organizaciones.

\section{EI CMI en la gestión de las organizaciones del sector público}

El modelo de "Nueva Gestión Pública" (NGP) ha motivado la aplicación en las entidades públicas de conceptos e instrumentos de gestión desarrollados y utilizados previamente en el ámbito privado. En concreto, el modelo de NGP provocó un creciente interés por la utilización de los sistemas de evaluación y gestión del rendimiento en las entidades públicas como instrumentos básicos para estimular y apoyar los cambios organizativos (HOOD, 1991, 1995; POLLITT y 
SUMMA, 1997; PALLOT, 1999; LAPSLEY, 1999; TER BOGT y VAN HELDEN, 2000; BLANCO et al., 2001; AIBAR, 2004; HOQUE, 2005; TER BOGT, 2008). En realidad, los últimos años han sido testigos de un creciente interés en mejorar la eficacia y la eficiencia en el sector público y, a su vez, de la generación de una inversión considerable en el desarrollo de medidas del desempeño. La gran cantidad de normas legales, los riesgos de su incumplimiento, la fijación de objetivos de forma vaga y amplia, y la escasez de recursos financieros colocan el sector público frente a la necesidad urgente de adoptar nuevos instrumentos de gestión, como es el caso del CMI, capaces de presentar objetivos consistentes con la estrategia delineada y suficientemente atractivos para apoyar a los gestores en sus propuestas junto de la alta dirección (CARMONA y GRÖNLUND, 2003; PEDRO, 2004).

Así, en los últimos años ha aumentado la popularidad y utilización del CMI entidades públicas y sin ánimo de lucro (BRIGNALL y MODELL, 2000; KLOOT y MARTIN, 2000; KAPLAN, 2001; JOHNSEN, 2001; RODRÍGUEZ y ORTIZ, 2002; CHAN, 2004; MODELL, 2004; WISNIEWSKI y ÓLAFSSON, 2004; LANG, 2004; BOCCI, 2005; RODRÍGUEZ et al., 2010; FÄLTHOLM y NILSSON, 2010; NILSSON, 2010; DEEM et al., 2010; SHARMA y GADENNE, 2011; HOQUE y ADAMS, 2011; MARIN, 2012).

En este sentido, según Kaplan y Norton (2004, p. 445), la aplicación del CMI en las organizaciones sin fines de lucro "ha sido una de las extensiones más gratificantes del concepto original”. Para dichos autores, estas organizaciones no tratan de generar resultados financieros por encima de la media sino de cumplir una determinada misión. Por consiguiente, necesitan de un sistema amplio de indicadores financieros y no financieros para motivar y evaluar su desempeño. Sin embargo, debe tenerse en cuenta que la aplicación de una práctica gerencial originada en el sector privado al ámbito de actuación de las entidades públicas no solo requiere que se realicen adaptaciones en el modelo original, sino, además, disponer de herramientas innovadoras propias (MORA y VIVAS, 2001). Dichas adaptaciones se refieren, fundamentalmente, al significado, denominación y peso relativo de las distintas perspectivas así como a las relaciones causales que se establecen entre ellas.

Como se puede observar en la Figura 1 existen dos campos de actuación central del CMI en el ámbito del sector público: uno de carácter interno, asociado al control político, y el otro de carácter externo, asociado a la comunicación política. Así, mientras que las empresas se rigen por los criterios de la efectividad y de la eficiencia, "las organizaciones públicas deben tener siempre en cuenta el aspecto de la legitimidad, pues desde el punto de vista teórico de la democracia existen grandes exigencias sobre la gestión pública con respecto a una actuación y a un comportamiento transparente y que se pueda justificar” (HORVÁTH \& PARTNERS, 2003, p. 450). 
Figura 1 - La doble función del CMI en el sector público

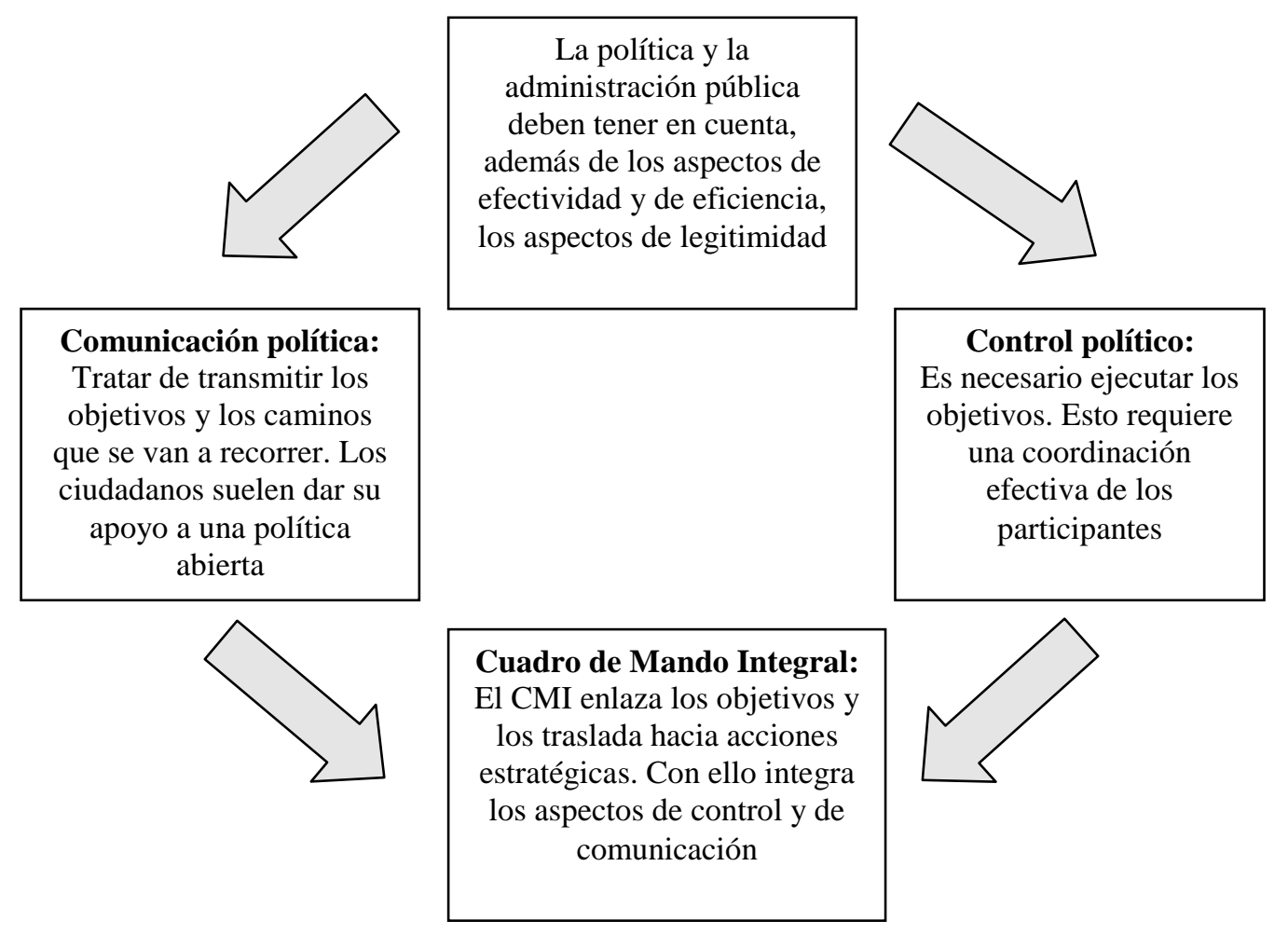

Fuente: Horváth \& Partners, 2003, p. 451.

En el caso concreto de la implementación del CMI en los ayuntamientos, y de acuerdo con Santos (2006), es fundamental la utilización de software específico y su integración con eventuales sistemas de apoyo a la decisión que sean utilizados, resaltando que existe un conjunto de características que distinguen los ayuntamientos de las empresas privadas y, consecuentemente, un conjunto de cuestiones previas y de factores críticos de éxito que se deben tener en consideración a la hora de implementar el CMI en dichas organizaciones. Estos aspectos se encuentran evidenciados en el cuadro siguiente.

Cuadro 1 - La implementación del CMI en los ayuntamientos

\begin{tabular}{||l||l||}
\hline \multicolumn{1}{|c|}{ Cuestiones Previas } & \multicolumn{1}{|c||}{ Factores Críticos de Éxito } \\
\hline \hline $\begin{array}{l}\text { ¿Cuál la misión, visión y valores que tendrán de ser } \\
\text { transcritos para el CMI para que la estrategia, a todos los } \\
\text { niveles, sea atingida? }\end{array}$ & $\begin{array}{l}\text { ¿Formar todos los usuarios, tanto a nivel individual } \\
\text { como eventualmente los equipos/grupos de trabajo a } \\
\text { quienes están cometidas responsabilidades específicas? }\end{array}$ \\
$\begin{array}{l}\text { ¿Quiénes van a ser los usuarios del CMI? Apenas las } \\
\text { autarcías? ¿El personal dirigente? ¿O también otros } \\
\text { niveles del ayuntamiento? }\end{array}$ & $\begin{array}{l}\text { Construir las métricas/indicadores estrictamente } \\
\text { necesarios, evitando el error común de tentar medir } \\
\text { demás, exigiendo un esfuerzo de análisis tan grande que } \\
\text { el CMI acaba por tornarse absolutamente impracticable } \\
\text { de utilizar, contribuyendo, así, para su descreencia; }\end{array}$ \\
¿Cómo va a ser utilizado? & \begin{tabular}{l} 
Empezar con un conjunto de métricas/indicadores \\
\hline
\end{tabular} \\
\hline
\end{tabular}


¿Cómo se va a tener acceso al CMI? ¿A través del sistema informático o en suporte papel para determinados usuarios? mínimo para que se posa comprobar la validad del sistema de CMI, y posteriormente ampliar a las restantes métricas/indicadores de una forma segura.

En el caso de una integración de los datos de otros sistemas con el sistema que suporta el CMI, ¿Cómo se garantiza la calidad/integridad de los datos?

¿Cómo se va a divulgar los resultados obtenidos?

Fuente: Santos, 2006, p. 175, adaptado.

Como observa Ferrão (2004), el CMI en los ayuntamientos tendrá impactos a nivel del aprendizaje (posibilitando el alineamiento de la estrategia en un contexto organizacional único y en un proceso de mejora continua, basado en sistemas de evaluación más objetivos y rigurosos), planificación (mejoras registradas en el proceso de elaboración y ejecución de presupuestos y asignación de recursos, al nortearse por medidas más realistas y objetivas, susceptibles de traducción en resultados mensurables y de facilitar el establecimiento de previsiones en cuanto al futuro), de los varios agentes implicados (las medidas anunciadas son más fácilmente evaluadas, aumentando la visibilidad de sus actividades y facilitando la participación de los distintos agentes en el proceso decisorio de los responsables del ayuntamiento), y a nivel del proceso de benchmarking (el CMI facilita la comparación del desempeño entre distintos ayuntamientos). Así, al tornar más visibles determinadas medidas, el CMI irá a facilitar la identificación de las mejores prácticas de gestión y la introducción de mejoras en los ayuntamientos.

\section{Las perspectivas del CMI en el sector público}

Coincidimos con Santos y Fidalgo (2003, p. 250) cuando afirman que "las organizaciones deben adoptar aquéllas perspectivas que mejor se adapten a sus características y, de esta forma, el CMI permitirá centrar la atención en aquellos factores clave específicos relacionados con la consecución de los objetivos estratégicos”. Así, de acuerdo con el tipo de organización y el entorno donde opera, la jerarquía de las perspectivas.

En relación a la perspectiva financiera del CMI, debe tenerse en cuenta que hay una notable diferencia entre los objetivos que persiguen las empresas privadas y los de las entidades públicas. Así, al contrario de lo que ocurre en las empresas privadas, en las que los objetivos financieros están en la cima de las relaciones de causa-efecto, ya que estas empresas persiguen fundamentalmente la maximización de su valor en el mercado, las organizaciones pertenecientes al sector público buscan sobre todo la maximización del bienestar de los ciudadanos. De allí que la perspectiva no figure en el primer lugar de la jerarquía, el cual pasa a ser ocupado por la perspectiva de clientes/ciudadanos. 
A este respecto, Mora y Vivas (2001) argumentan que en las entidades públicas los resultados financieros, aunque necesarios e importantes, deben complementarse con muchos otros, en la medida en que lo que se pretende realmente optimizar son los servicios prestados a los ciudadanos y la satisfacción de las necesidades de distintos grupos de interés, quienes a la vez son electores de los responsables públicos. En efecto, en las entidades públicas y sin fines de lucro es esta misión y no los objetivos financieros o de los accionistas lo que guía la estrategia organizativa (CARVALHO y AZEVEDO, 2001; RUAS, 2003).

En definitiva, en las organizaciones del sector público la perspectiva financiera constituye un medio para lograr un fin y no un fin en sí mismo. Por consiguiente, al contrario de lo que ocurre en el ámbito privado, la perspectiva financiera no es el foco al que se orientan las restantes perspectivas. Sin embargo, debe tenerse en cuenta que ningún scorecard queda completo si no incluye esta perspectiva, si bien con una importancia más reducida, ya que cualquier organización necesita contar con recursos financieros para funcionar con éxito y satisfacer sus clientes (PINTO, 2007). En este sentido, en el ámbito público, la perspectiva financiera se orienta básicamente al mantenimiento del equilibrio presupuestario, planteando objetivos de reducción de costes y de eficiencia (AIDEMARK, 2001; KAPLAN, 2001; ELLINGSON y WAMBSGANSS, 2001; MORA y VIVAS, 2001; RUAS, 2003; HORVÁTH \& PARTNERS, 2003; SILVA FILHO y RODRÍGUEZ, 2004; BORONAT, 2004). Para Davila (1999), una vez que no es el objetivo primordial, la perspectiva financiera debería ser sustituida por una perspectiva denominada "recursos". En el mismo sentido, Walsh (2000) sugiere la designación “Administración, Reducción de Costes o Recursos" para dicha perspectiva.

Por otro lado, en opinión de Mora y Vivas (2001, p. 77) al tiempo que el peso de la perspectiva financiera se reduce considerablemente en las entidades públicas, debe incrementarse el peso de la perspectiva de los clientes/ciudadanos, puesto que "la razón de ser de los organismos públicos radica en la necesidad de proveer servicios a los ciudadanos que el mercado nunca proveería, o en todo caso lo haría a precios no razonables". Por consiguiente, en las organizaciones del sector público la perspectiva de clientes/usuarios pasa a ser la dominante, en detrimento de la perspectiva financiera, ya que el objetivo final de estas organizaciones es la satisfacción de los usuarios de los servicios públicos en lo que respecta a la calidad del servicio prestado. Por otra parte, Kloot y Martin (2000), Walsh (2000) y Silva Filho y Rodríguez (2004) defienden la sustitución de la perspectiva de clientes por una perspectiva que denominan de la comunidad, en la que se contemplan aspectos relativos a la habilidad de la entidad para proporcionar bienes y servicios de calidad, la efectividad con la que éstos son ofrecidos y la atención al cliente así como el impacto de la actuación de la entidad sobre el conjunto de la comunidad en general. Por consiguiente, podemos afirmar que, además de incrementar su importancia, en las entidades 
públicas y sin fines de lucro, la perspectiva del cliente amplia su alcance, incluyendo a un mayor abanico de stakeholders (TER BOGT y VAN HELDEN, 2000; SANTOS y FIDALGO, 2004).

En lo que respecta a la perspectiva de procesos internos, a semejanza de lo que ocurre en las empresas privadas, en las entidades públicas esta perspectiva debe reflejar los factores críticos de éxito (procesos y competencias) con que cuenta la organización para lograr la calidad de los servicios y aumentar la percepción de valor por parte del cliente/usuario/ciudadano en relación con los bienes y servicios ofrecidos por la entidad, Es decir, aumentar el denominado value for money o valor a cambio de gasto (en este caso, los impuestos y/o las tasas públicas). Por otro lado, según Kaplan y Norton (2001), los cuadros de mando iniciales de las entidades públicas tienden a centrarse en los aspectos considerados dentro de la perspectiva de procesos internos: tiempo (duración), calidad y coste (productividad) de los procesos.

Finalmente, la perspectiva de aprendizaje y crecimiento asume una especial relevancia en el sector público, una vez que, para alcanzar las metas fijadas, estas entidades dependen fuertemente de las capacidades, motivación, dedicación, capacidad de delegación e implicación de sus recursos humanos (MORA y VIVAS, 2001; RUAS, 2003; PINTO, 2007). Por consiguiente, en las organizaciones públicas, la perspectiva del personal o de los recursos humanos es aún más explícita, con un énfasis en un mayor número de stakeholders (CARMONA y GRÖNLUND, 2003; AX y BJORNENAK, 2005; MODELL, 2009).

En opinión de Kaplan y Norton (2001), el cambio en la jerarquía de las perspectivas del CMI que acabamos de describir representa la principal modificación en lo que respecta a la implantación del CMI en el sector público (Figura 2).

Figura 2 - Las perspectivas del CMI y la misión de las entidades del sector público

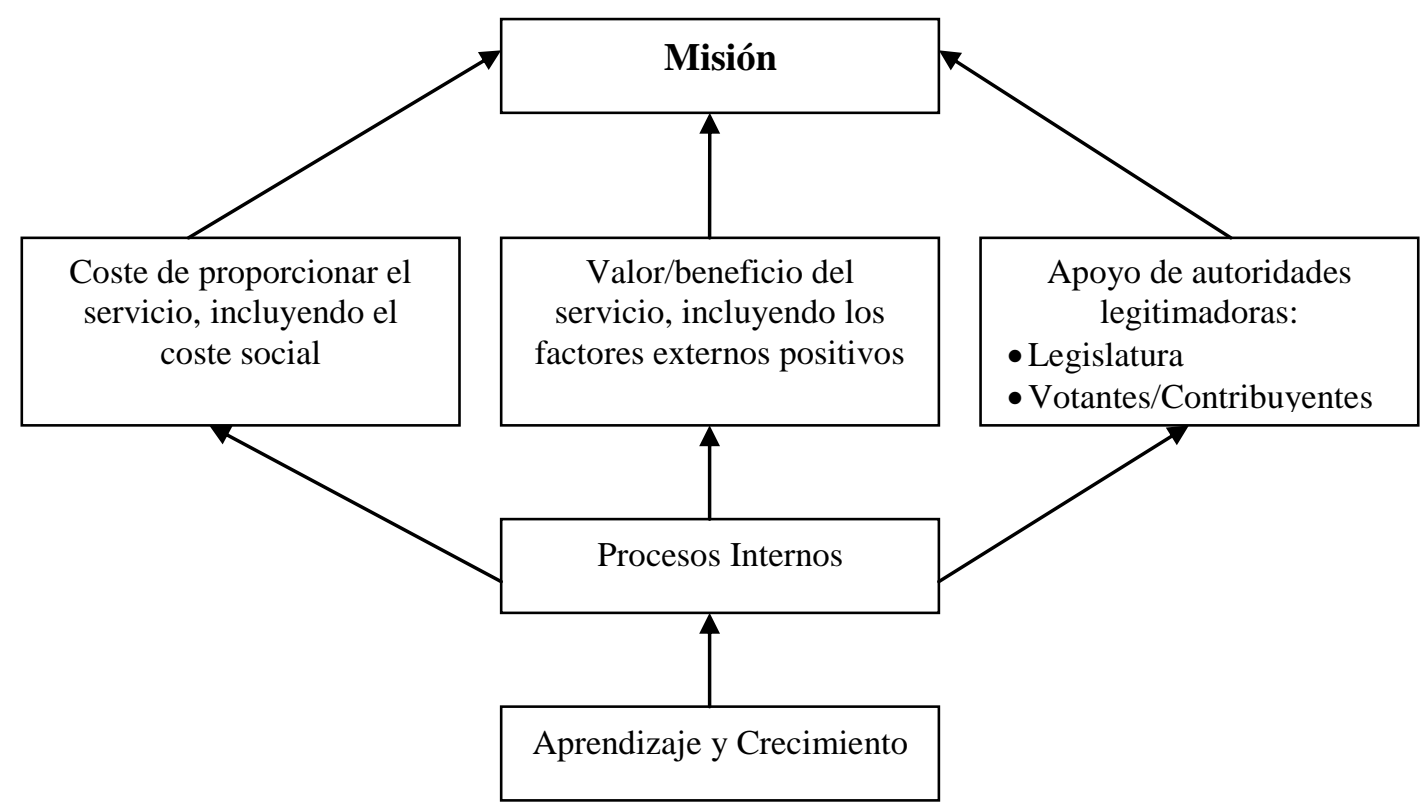

Fuente: Kaplan y Norton, 2001, p. 149. 
A este respecto, Voelker et al. (2001) plantean una representación piramidal de las perspectivas del CMI en entidades no lucrativas, donde la perspectiva de aprendizaje y crecimiento constituye la base de la pirámide. El siguiente nivel de la pirámide es la perspectiva de los procesos internos, seguida de la perspectiva financiera. Finalmente, en el nivel más alto de la pirámide estaría la perspectiva de clientes o stakeholders. Por otra parte, Yetano (2005) sugiere la siguiente ordenación de perspectivas:

- Comunidad: refleja la multiplicidad de interesados/afectados por las actividades de la entidad pública (ciudadanos, empresas, asociaciones, medios de comunicación, tribunales de cuentas y otros organismos públicos). Su objetivo es medir el nivel de satisfacción que generan los servicios prestados, promoviendo la transparencia, la justicia y la responsabilización (accountability);

- Procesos internos: refleja las operaciones consideradas críticas para la consecución de los objetivos, enfatizando la innovación, la calidad y los costes de las actividades y procesos operativos desarrollados;

- Aprendizaje y crecimiento: refuerza la gestión de las tareas vinculadas al binomio formación y aprendizaje (una de las debilidades de las administraciones locales), es decir, el fomento y potenciación de las habilidades y dedicación del personal. En palabras de Yetano (2005, p. 42), “el objetivo será fortalecer aspectos como el ambiente y la cultura organizacional, la tecnología y los sistemas de información y, sobre todo, dar al personal la formación adecuada (...)";

- Presupuestos: refleja los recursos financieros para lograr los objetivos de la comunidad.

\section{Modelos basados en el CMI desarrollados específicamente para el sector público}

\subsection{El modelo de Niven (2003)}

Niven (2003) presenta un modelo de CMI para el sector público (Figura 3) en el que coloca la misión en la cima del CMI y mantiene la estrategia en el centro del CMI. En cuanto a las distintas perspectivas, considera que la perspectiva de clientes es fundamental para el cumplimiento de la misión y, por tanto, ocupa un lugar destacado frente a las restantes perspectivas. En este sentido, como apuntaron anteriormente Kaplan y Norton (2001, p. 174), la diferencia principal entre estos sectores "ha sido una consideración más cuidadosa de los clientes. Los clientes se ven elevados a la parte más alta de los mapas estratégicos de un CMI, dado que, en definitiva, la prestación efectiva de servicios a los clientes explica la existencia de la mayoría de Administraciones Públicas y no lucrativas". 
Figura 3 - El CMI en el sector público

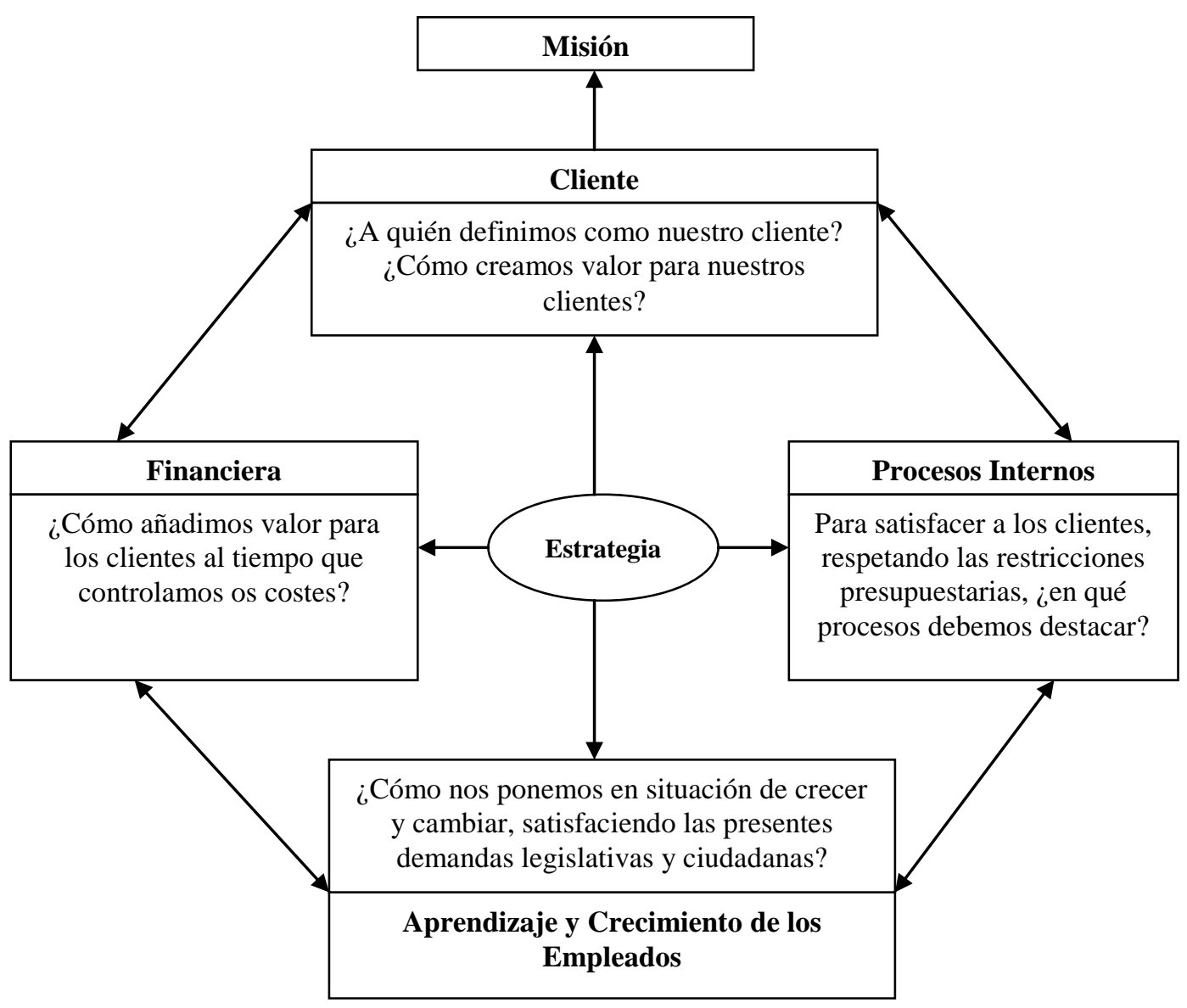

Fuente: Niven, 2003, p. 380.

\subsection{El modelo de Olve et al. $(1999,2002)$}

Olve et al. (1999, 2002) analizaron ejemplos de las perspectivas adoptadas por diferentes ayuntamientos suecos y concluyeron que, aunque las experiencias eran análogas a las de algunas empresas privadas, era necesario efectuar algunas adaptaciones y modificaciones en el modelo original (figura 4). En concreto, planteaban:

1. Sustituir la perspectiva financiera;

2. Cambiar el foco de atención en el cliente a las relaciones con el comercio y las asociaciones locales, proveedores y ciudadanos;

3. Tener en cuenta el hecho que en la definición de prioridades, puede ser conveniente, en términos políticos, evitar la claridad requerida por el CMI.

Figura 4 - Generalización del modelo de CMI para el sector público 


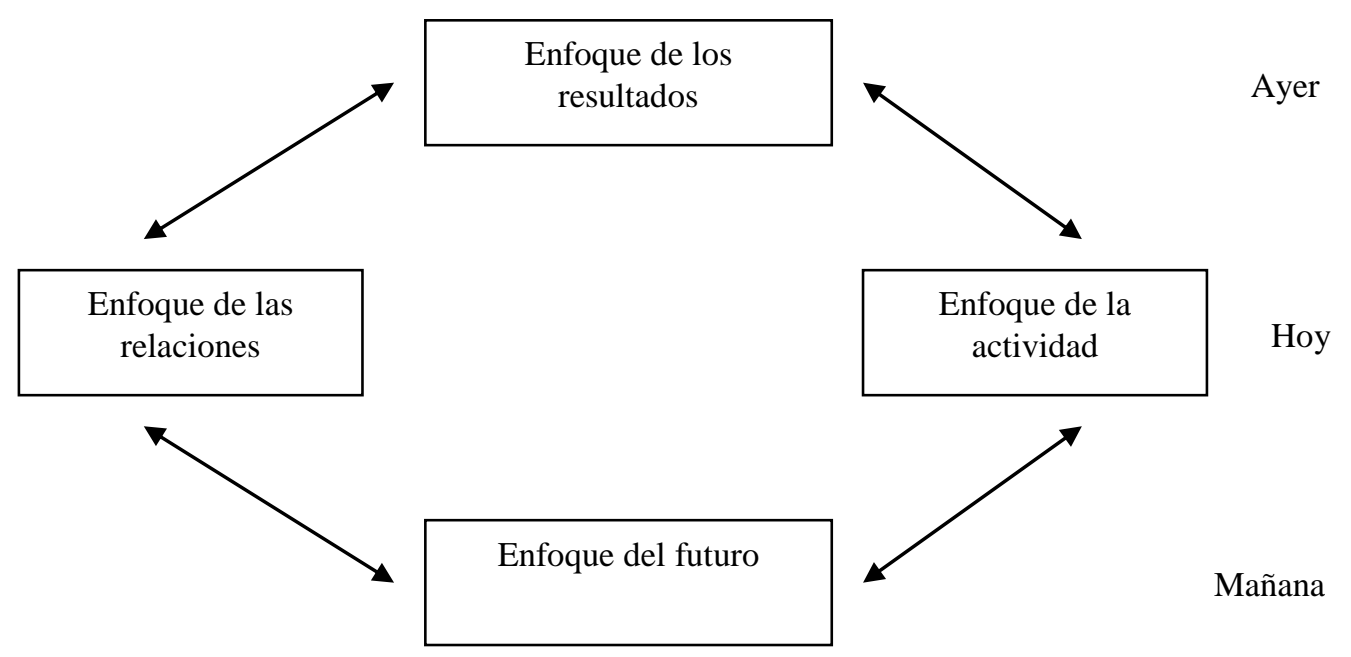

Fuente: Olve et al., 2002, p. 135.

\subsection{El modelo de Bastidas y Ripoll (2003)}

En opinión de Bastidas y Ripoll (2003), para que el CMI constituya un modelo viable en las instituciones públicas es necesario que las perspectivas del usuario, de la comunidad (considerada como un participante activo y directo en la formulación de las prioridades estratégicas de las entidades públicas y como fiscalizadores de su actuación al comprobar que la organización está respondiendo a sus expectativas) y medioambiental (referida a la responsabilidad social con la comunidad para velar por la protección del medio ambiente) ocupen posiciones paralelas en la estructura jerárquica del CMI (dada su importancia en el establecimiento de prioridades estratégicas) y que la perspectiva de aprendizaje y crecimiento sea sustituida por la que denominan perspectiva humana, con objetivos concernientes a la propuesta de valor para los trabajadores y gerentes, fomentando la motivación, el rendimiento y la participación de los miembros de la organización (Figura 5).

Además, dichos autores proponen como ajustes al modelo del CMI para las entidades públicas: (1) la integración de la planificación estratégica como un proceso indispensable para la identificación de los stakeholders, sus intereses, de conflictos potenciales que se pueden generar y de la influencia de las relaciones de poder; (2) la consideración de la perspectiva financiera como un medio para alcanzar los fines de las demás perspectivas y (3) la consideración de la entrega de valor a los proveedores como una variable clave, así como la comparación con sus rivales inmediatos, como un método para impulsar la mejora y la innovación. 
Figura 5 - Ajustes propuestos al modelo del CMI para las entidades públicas

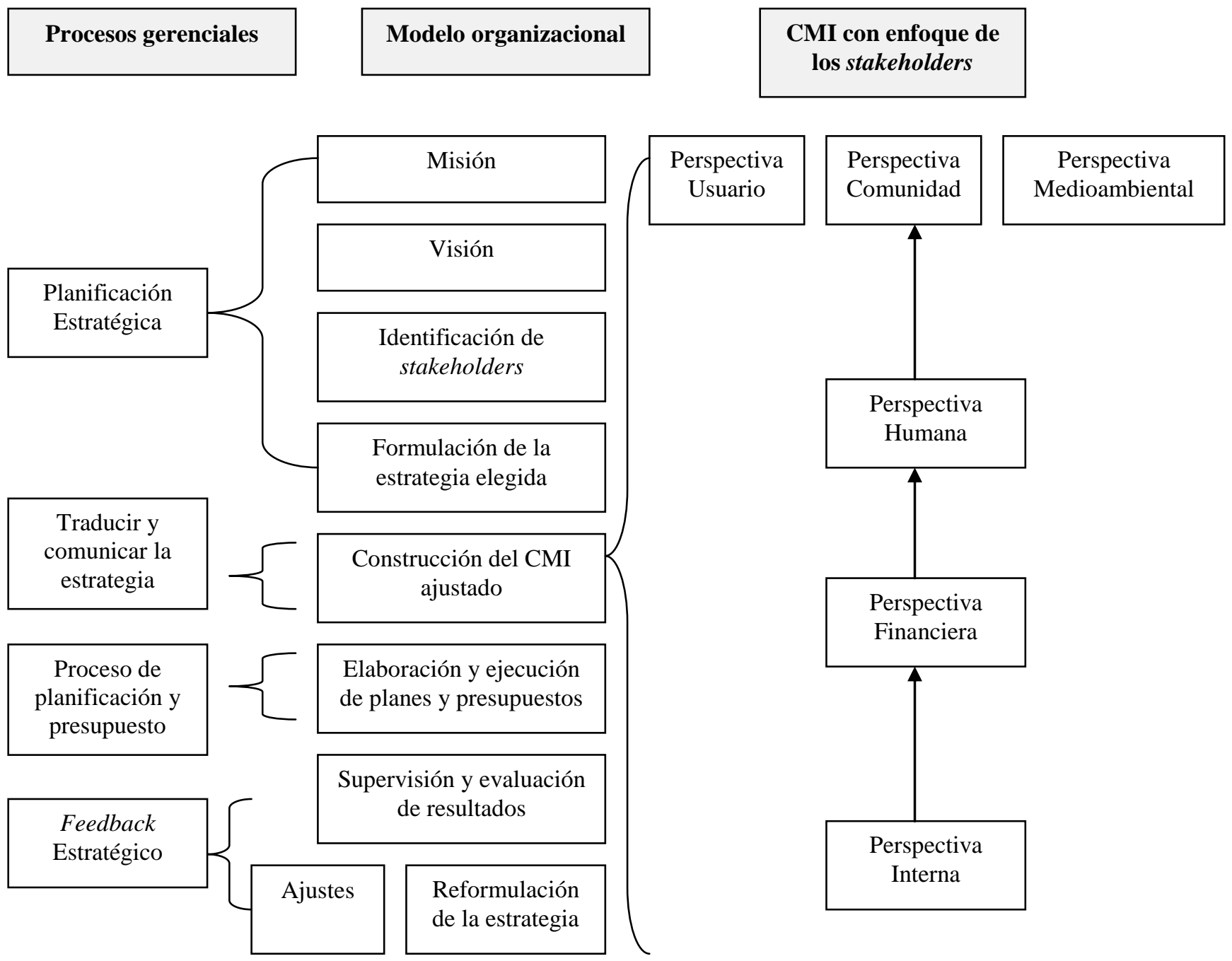

Fuente: Bastidas y Ripoll, 2003, p. 39.

\section{Ventajas y aspectos críticos en la implantación del CMI en el sector público}

La importancia de la aplicación del CMI en entidades públicas y sin ánimo de lucro ha sido subrayada con frecuencia en la literatura. Así, para Mora y Vivas (2001), el CMI permite introducir formalmente en la Administración Pública un factor que tradicionalmente no se tenía en cuenta: la planificación estratégica y la posibilidad de utilizar variables no financieras para evaluar el rendimiento. Además, el CMI representa un cambio cultural importante por lo que respecta al ocultismo informativo y permite "romper con la planificación determinista que representan los mandatos políticos (estrategias solo para cuatro años) y vincular los programas de gobierno a la consecución de objetivos planificados en un tiempo razonable, con calidad" (MORA y VIVAS, 2001, p. 77). Por otro lado, Rodríguez y Ortiz (2002) resumen la importancia del CMI en las entidades públicas en dos aspectos fundamentales: (1) el hecho de que a través de un conjunto de 
inductores de actuación permite traducir la estrategia de la entidad en relaciones de causa-efecto y (2) su potencial para promover el que la organización apueste por los activos intangibles como motor para la prestación de bienes y servicios dirigidos a la satisfacción de las necesidades de la comunidad.

En resumen, como ventajas de la utilización del CMI en la gestión pública podemos citar (ATKINSON y EPSTEIN, 2000; AIDEMARK, 2001; KAPLAN, 2001; NIVEN, 2003; BASTIDAS y RIPOLL, 2003; PEDRO, 2003, 2004; BORONAT, 2004; WISNIEWSKI y ÓLAFSSON, 2004; SANTOS, 2006; BASTIDAS y MORENO, 2006; LAWSON et al., 2007; WOODS y GRUBNIC, 2008; RAUTIAINEN, 2009; FÄLTHOLM y NILSSON, 2010):

- Obliga a concretar y articular la estrategia de la organización;

- Proporciona una herramienta de mejora continua y revisión de la estrategia;

- Permite establecer los objetivos de una forma más coherente, facilitando la alineación de los objetivos con la estrategia;

- Traduce la estrategia y la misión de la organización en indicadores que informan sobre el grado de consecución de los objetivos;

- Agiliza y coordina la comunicación de la estrategia a toda la organización, creándose un lenguaje común en la misma;

- Integra el corto, medio y largo plazo;

- Ayuda a gestionar el cambio (puede ser una herramienta eficaz para introducir cambios más duraderos en las organizaciones del sector público, para que puedan responder a las demandas de su entorno social, económico y político);

- Cubre la totalidad de las operaciones (integra toda las áreas de la sociedad y de la Administración);

- Define los factores críticos de éxito;

- Permite la adopción rápida y correcta de decisiones relativas a distintos ámbitos;

- Otorga fortaleza al sistema de información de la entidad;

- Permite analizar posiciones frente a presupuestos, ejercicios anteriores y distintos meses;

- Posibilita una visión gráfica de la Administración;

- Reduce el grado de incertidumbre y favorece el desarrollo de organizaciones públicas más eficientes;

- Promueve una cultura basada en la gestión por objetivos.

Frente a las ventajas señaladas, los principales problemas que entraña la aplicación del CMI en el sector público tienen que ver con las dificultades asociadas a la definición de los clientes de las entidades públicas y a las derivadas de las peculiaridades propias de dichas entidades. Así, para 
Blanco et al. (2007), el hecho de que la figura del cliente en las entidades públicas no esté claramente delimitada, hace difícil y compleja la tarea de conocer sus expectativas con relación a los servicios que se suministran. Además, los resultados de los servicios públicos no pueden definirse mediante outputs únicos, lo que plantea la necesidad de distinguir entre los servicios proporcionados por la entidad pública a los ciudadanos y el impacto que tienen dichos servicios sobre el conjunto de la sociedad. Por otro lado, Horváth \& Partners (2003) resalta la dificultad existente para documentar las relaciones causa-efecto en las entidades públicas y el hecho de que sus objetivos rara vez son consistentes, en la medida en que dependen de un proceso de negociación muchas veces influido por cuestiones políticas. Por su parte, Micheli y Kennerley (2005) señalan también la dificultad para introducir una cultura de medición en las organizaciones públicas.

En resumen, la elevada dificultad para definir y medir los resultados y relacionarlos con los objetivos, la multiplicidad de stakeholders (con demandas divergentes e incluso, en algunos casos, contradictorias), la resistencia al cambio, la falta de iniciativa política, la ausencia de apoyo de los ejecutivos y entidades superiores, la falta del tiempo necesario para su desarrollo e implantación, la falta de recompensas asociadas al desempeño, la falta de formación de los recursos humanos y la limitación de recursos son algunos de los principales factores que dificultan la implementación de este tipo de modelos de evaluación y gestión del rendimiento en las entidades públicas (GOMES et al., 2007).

\section{Estudio empírico}

\subsection{Objetivos}

Como hemos comentado con anterioridad, además de proporcionar algunas ideas acerca de la importancia, las características y las necesarias adaptaciones del CMI para su aplicación a organizaciones públicas y sin ánimo de lucro, este trabajo pretende obtener evidencia empírica sobre el conocimiento y utilización del CMI en los ayuntamientos portugueses, así como identificar, a través de un análisis descriptivo, las principales razones inherentes a la no adopción o abandono del CMI en dichas organizaciones.

\subsection{Metodología}

Como medio básico para la recolección de los datos hemos optado por la realización de una encuesta postal a través del envío de un cuestionario a todos los ayuntamientos portugueses (308 ayuntamientos).

Los cuestionarios fueron testados durante el mes de marzo de 2009 por elementos pertenecientes a la población a investigar así como por académicos conocedores del tema en estudio 
y con amplia experiencia en el diseño y realización de investigaciones a través de encuestas, y han sido dirigidos al presidente del ayuntamiento. Decidimos dirigir los cuestionarios a estos responsables por considerarlos los más adecuados debido a su papel a nivel de la toma de decisiones sobre la estrategia y gestión de las organizaciones así como por su conocimiento de la información sobre la cual pretendíamos obtener evidencia. Sin embargo, en todos los casos se solicitaba que el cuestionario se entregara a la persona de la organización que se considerara más indicada para responder a las cuestiones planteadas.

La fase de recogida de la información tuvo lugar entre abril de 2009 y marzo de 2010 y los datos recogidos se han tratado estadísticamente con recurso al programa SPSS (Statistical Package for the Social Sciences - versión 17). De los 308 cuestionarios enviados obtuvimos 100 respuestas, lo que corresponde al $32,5 \%$ del total de la población. A medida que se iban recibiendo los cuestionarios, éstos eran objeto de revisión con el propósito de identificar "valores en falta" (missing values) resultantes de errores al rellenarlo o de no haberse respondido a alguna(s) pregunta(s). Tras dicho análisis, uno de los 100 cuestionarios recibidos fue considerado no válido, por presentar datos muy incompletos. En consecuencia, disponemos de 99 cuestionarios válidos.

\subsection{Resultados}

\subsubsection{Caracterización general de las organizaciones de la muestra}

\section{a) Localización geográfica}

En lo que respecta a la localización geográfica de los ayuntamientos que integran la muestra objeto de estudio, hemos organizado las respuestas de acuerdo con las siete regiones geográficas del país que componen las NUTS II (sigla utilizada para designar en términos estadísticos las unidades de según nivel de la Nomenclatura de las Unidades Territoriales). Como puede verse en el Cuadro 2, la mayoría de los ayuntamientos de la muestra se encuentran situados en la región Norte (38) y Centro (26) del país y solo siete ayuntamientos pertenecen a las regiones autónomas (5 en las Azores y 2 en Madeira). Dado que la prueba de ajuste de Chi-cuadrado presenta un estadístico no significativo ( $p$-value $=0,319$ ), la muestra puede considerarse representativa de la población en términos de localización geográfica. 
Cuadro 2 - Distribución de los ayuntamientos por localización geográfica

\begin{tabular}{||l||c||c||c||c||c|c||}
\hline \multicolumn{1}{|c||}{ Región Geográfica } & \multicolumn{2}{c||}{ Respuestas } & \multicolumn{2}{c||}{ No Respuestas } & \multicolumn{2}{c||}{ Total } \\
\cline { 2 - 7 } & $\mathbf{N}$ & $\mathbf{\%}$ & $\mathbf{N}$ & $\mathbf{\%}$ & $\mathbf{N}$ & \% \\
\hline \hline Norte & 38 & 38,4 & 48 & 23 & 86 & 27,9 \\
\hline \hline Centro & 26 & 26,3 & 51 & 24,4 & 77 & 25 \\
\hline \hline Lisboa y Valle del Tajo & 13 & 13,1 & 38 & 18,2 & 51 & 16,6 \\
\hline \hline Alentejo & 11 & 11,1 & 36 & 17,2 & 47 & 15,3 \\
\hline \hline Algarbe & 4 & 4 & 13 & 6,2 & 17 & 5,5 \\
\hline \hline Azores & 5 & 5,1 & 14 & 6,7 & 19 & 6,2 \\
\hline Madeira & 2 & 2 & 9 & 4,3 & 11 & 3,6 \\
\hline \hline \multicolumn{1}{|c|}{ Total } & $\mathbf{9 9}$ & $\mathbf{1 0 0}$ & $\mathbf{2 0 9}$ & $\mathbf{1 0 0}$ & $\mathbf{3 0 8}$ & $\mathbf{1 0 0}$ \\
\hline
\end{tabular}

$\left(p\right.$-value $\left.=0,319 ; \chi^{2}=7,023\right)$

Fuente: Pesquisa de campo (2010).

\section{b) Dimensión}

Como posibles indicadores de la dimensión de los ayuntamientos hemos utilizado el volumen de ingresos, el volumen de gastos, el número de habitantes y el número de trabajadores, todos relativos al año 2008. En cuanto al volumen de ingresos y de gastos (Cuadro 3) es posible constatar que la mayoría de los ayuntamientos que integran la muestra posee un volumen de ingresos y de gastos inferior a $20.000 .000 €$ (62 y 63 ayuntamientos, respectivamente) y solo dieciséis ayuntamientos (el 16,3\%) presentan valores superiores a 50.000.000€.

Cuadro 3 - Volumen de ingresos y de gastos de los ayuntamientos

\begin{tabular}{|c|c|c|c|c|}
\hline \multirow[t]{2}{*}{ Valores (€) } & \multicolumn{2}{|c|}{ Volumen de Ingresos } & \multicolumn{2}{|c|}{ "Volumen de Gastos } \\
\hline & Ayuntamientos & Porcentaje & Ayuntamientos & Porcentaje \\
\hline Menos de 10.000.000 & 28 & 28,6 & 26 & 26,5 \\
\hline Entre 10.000 .000 y 20.000 .000 & 34 & 34,7 & 37 & 37,8 \\
\hline Entre 20.000 .000 y 30.000 .000 & 13 & 13,3 & 11 & 11,2 \\
\hline Entre $30.000 .000 \mathrm{y} 40.000 .000$ & 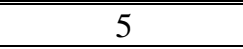 & 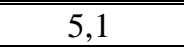 & 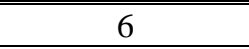 & 6,1 \\
\hline Entre 40.000 .000 y 50.000 .000 & 2 & 2 & 2 & 2 \\
\hline Más de 50.000.000 & 16 & 16,3 & 16 & 16,3 \\
\hline Total & 98 & 100 & 98 & 100 \\
\hline
\end{tabular}

Fuente: Pesquisa de campo (2010).

Con respecto al número de habitantes (Cuadro 4), y de acuerdo con la clasificación de Carvalho et al. (2009), el 59,6\% de los ayuntamientos son de pequeña dimensión (población menor o igual a 20.000 habitantes), el 30,3\% de los ayuntamientos puede caracterizarse como de mediana dimensión (población mayor que 20.000 habitantes y menor o igual a 100.000 habitantes) y solo el $10,1 \%$ de los ayuntamientos pueden ser considerados de gran dimensión (población superior a 100.000 habitantes). 
Cuadro 4 - Número de habitantes de los ayuntamientos

\begin{tabular}{||c||c||c|}
\hline \multicolumn{1}{|c|}{ Habitantes } & Ayuntamientos & Porcentaje \\
\hline \hline Menor o igual a 20.000 & 59 & 59,6 \\
\hline \hline Entre 20.000 y 100.000 & 30 & 30,3 \\
\hline \hline Mayor que 100.000 & 10 & 10,1 \\
\hline \hline Total & $\mathbf{9 9}$ & $\mathbf{1 0 0}$ \\
\hline
\end{tabular}

Fuente: Pesquisa de campo (2010).

Finalmente, en lo que concierne al número de trabajadores (Cuadro 5), la casi totalidad de los ayuntamientos de la muestra (68 ayuntamientos) tiene hasta 500 trabajadores y, de ellos, la mayoría (el 52,3\%) tiene hasta 250 trabajadores. Los ayuntamientos con mayor número de empleados, más de 500, suponen el 22,7\% (20 ayuntamientos).

Cuadro 5 - Número de trabajadores de los ayuntamientos

\begin{tabular}{|l|c|c|}
\hline \multicolumn{1}{|c|}{ Trabajadores } & Ayuntamientos & Porcentaje \\
\hline \hline Menos de 250 & 46 & 52,3 \\
\hline \hline $250-500$ & 22 & 25 \\
\hline \hline $500-1.000$ & 10 & 11,4 \\
\hline \hline Más de 1.000 & 10 & 11,4 \\
\hline \hline \multicolumn{1}{|c|}{ Total } & $\mathbf{8 8}$ & $\mathbf{1 0 0}$ \\
\hline
\end{tabular}

Fuente: Pesquisa de campo (2010).

\section{c) Certificación}

Los siguientes cuadros muestran el número y porcentaje de ayuntamientos de la muestra que cuentan con una certificación de la calidad y/o medioambiental así como aquéllos que elaboran informes de sostenibilidad y/o medioambientales. Como puede observarse, la mayoría de los ayuntamientos de la muestra (el 58,6\%) no están certificados en términos de la calidad y la casi totalidad (el 92,9\%) no posee certificación medioambiental. Los ayuntamientos que indicaron estar certificados obtuvieron la certificación en base a las normas ISO 9001 (año 2000 y año 2008), ISO 14001 y OHSAS 18001. Por otro lado, solo un número reducido de ayuntamientos elaboran informes de sostenibilidad y/o medioambientales (el 10,1\%).

Cuadro 6 - Certificación de la calidad y medioambiental de los ayuntamientos

\begin{tabular}{||c||c|c||c|c||}
\hline \multicolumn{1}{|c||}{} & \multicolumn{2}{c||}{ Certificación de la calidad } & \multicolumn{2}{c||}{ Certificación medioambiental } \\
\cline { 2 - 5 } & Ayuntamientos & Porcentaje & Ayuntamientos & Porcentaje \\
\hline \hline Sí & 29 & 29,3 & 5 & 5,1 \\
\hline \hline No & 58 & 58,6 & 92 & 92,9 \\
\hline \hline En curso & 12 & 12,1 & 2 & 2 \\
\hline \hline \multicolumn{1}{|c|}{ Total } & $\mathbf{9 9}$ & $\mathbf{1 0 0}$ & $\mathbf{9 9}$ & $\mathbf{1 0 0}$ \\
\hline
\end{tabular}

Fuente: Pesquisa de campo (2010). 
Cuadro 7 - Elaboración de informes de sostenibilidad y/o medioambientales en los ayuntamientos

\begin{tabular}{|l|c||c|}
\hline & Ayuntamientos & Porcentaje \\
\hline \hline Sí & 10 & 10,1 \\
\hline \hline No & 89 & 89,9 \\
\hline \hline Total & $\mathbf{9 9}$ & $\mathbf{1 0 0}$ \\
\hline
\end{tabular}

Fuente: Pesquisa de campo (2010).

\subsubsection{Caracterización general del mercado y el entorno organizativo}

En lo que respecta a las características del mercado y el entorno organizativo, los datos obtenidos indican que la opción más señalada por los encuestados fue que existen "algunos" niveles jerárquicos (61 ayuntamientos).

En cuanto al grado de concentración de la toma de decisiones en la alta dirección, observamos que el proceso de toma de decisiones está muy centralizado o centralizado en la alta dirección (72 ayuntamientos). Por otro lado, como puede observarse en el Cuadro 8, debe subrayarse la existencia de un sistema de reglas formales y la división del trabajo se realiza en base a la especialización y funcionalización, con valores medios superiores.

Cuadro 8 - Formalización/burocratización en los ayuntamientos

\begin{tabular}{||l|c||c||}
\hline \multicolumn{1}{|c|}{ Formalización/burocratización (n=98) } & Media & Desviación típica \\
\hline \hline División del trabajo en base a la especialización y funcionalización & 3,32 & 0,652 \\
\hline \hline Existencia de un sistema de reglas formales & 3,23 & 0,771 \\
\hline \hline Gestión/relaciones informales & 3,14 & 0,689 \\
\hline \hline $\begin{array}{l}\text { Utilización de procedimientos operativos estándar en la gestión del trabajo } \\
\text { realizado en los diferentes departamentos/ unidades/ secciones/ divisiones }\end{array}$ & 3,06 & 0,771 \\
\hline \hline $\begin{array}{l}\text { Formalización en la descripción de las actuales especificaciones de las tareas de } \\
\text { trabajo y de los criterios de evaluación del desempeño en la organización }\end{array}$ & 3,04 & 0,731 \\
\hline
\end{tabular}

Fuente: Pesquisa de campo (2010).

En cuanto a la previsibilidad/imprevisibilidad del entorno de las organizaciones (Cuadro 9), los encuestados consideran que el entorno está en constante cambio. Debe notarse también que muchos encuestados coinciden con las afirmaciones de que "el entorno de la organización es generalmente previsible" y de que "es difícil prever el impacto que tendrá en la organización una decisión incorrecta o la forma en que los factores del entorno pueden afectar al éxito o fracaso de una decisión”.

Cuadro 9 - Grado de previsibilidad/imprevisibilidad del entorno interno y externo de los ayuntamientos

\begin{tabular}{||l||c|c||}
\hline \multicolumn{1}{|c|}{ Entorno interno y externo (n=98) } & Media & Desviación típica \\
\hline \hline El entorno de la organización está en constante cambio & 3,70 & 0,911 \\
\hline \hline El entorno de la organización es generalmente previsible & 3,27 & 0,832 \\
\hline \hline Las previsiones sobre el entorno son generalmente precisas & 3,07 & 0,790 \\
\hline \hline $\begin{array}{l}\text { Los problemas generados por el entorno de la organización generalmente se } \\
\text { caracterizan como rutinarios y pueden ser enfrentados por procedimientos } \\
\text { corporativos establecidos }\end{array}$ & 2,92 & 0,893 \\
\hline $\begin{array}{l}\text { Existe falta de información respecto a los factores del entorno externo que afectan al } \\
\text { proceso de toma de decisiones }\end{array}$ & 3,01 & 0,925 \\
\hline
\end{tabular}




\begin{tabular}{|l|c|c||}
\hline $\begin{array}{l}\text { Es difícil prever el impacto que puede tener en la organización una decisión } \\
\text { incorrecta }\end{array}$ & 3,08 & 0,938 \\
\hline $\begin{array}{l}\text { Es difícil prever cómo los factores del entorno interno y externo pueden afectar al } \\
\text { éxito o fracaso de una decisión }\end{array}$ & 3,22 & 0,831 \\
\hline \hline
\end{tabular}

Fuente: Pesquisa de campo (2010).

En lo que concierne a las características de la cultura organizativa (Cuadro 10), los encuestados, por regla general, están en desacuerdo con la caracterización de la organización como conservadora y adversa al riesgo y con la afirmación de que "un estilo de gestión autoritario y una cultura burocrática pueden facilitar el proceso de adopción, implementación y uso de técnicas de Contabilidad de Gestión en la organización". Los resultados obtenidos evidencian que las organizaciones consideran que un estilo de gestión más participativo y consultivo facilita el proceso de adopción, implementación y uso de técnicas de Contabilidad de Gestión en la organización.

Cuadro 10 - Características de la cultura organizativa en los ayuntamientos

\begin{tabular}{||l||c||c||}
\hline \multicolumn{1}{|c|}{ Cultura organizativa (n=98) } & Media & Desviación típica \\
\hline \hline Los empleados dudan en expresar su desacuerdo con sus superiores & 3,01 & 0,984 \\
\hline \hline La mayoría de las decisiones operativas son tomadas en la alta dirección & 3,60 & 0,894 \\
\hline \hline La organización se caracteriza por ser conservadora y adversa al riesgo & 2,85 & 0,998 \\
\hline \hline $\begin{array}{l}\text { En la organización existe una clara comunicación, asistencia y apoyo a los } \\
\text { trabajadores }\end{array}$ & 3,27 & 0,864 \\
\hline \hline $\begin{array}{l}\text { La organización promueve la discusión de cuestiones estratégicas y la creatividad } \\
\text { de sus miembros }\end{array}$ & 3,01 & 0,856 \\
\hline \hline $\begin{array}{l}\text { En la organización existe un elevado intercambio de información, promoviéndose } \\
\text { la cooperación funcional }\end{array}$ & 3,20 & 0,979 \\
\hline \hline Las sugerencias de los empleados son valoradas & 3,42 & 0,994 \\
\hline \hline Existe un clima organizacional favorable al crecimiento organizativo y personal & 3,18 & 0,901 \\
\hline \hline $\begin{array}{l}\text { Un estilo de gestión autoritario y una cultura burocrática pueden facilitar el } \\
\text { proceso de adopción, implementación y uso de técnicas de Contabilidad de } \\
\text { Gestión en la organización }\end{array}$ & 2,13 & 0,820 \\
\hline \hline $\begin{array}{l}\text { Un estilo de gestión más participativo y consultivo facilita el proceso de adopción, } \\
\text { implementación y uso de técnicas de Contabilidad de Gestión en la organización }\end{array}$ & 4,13 & 0,652 \\
\hline \hline
\end{tabular}

Fuente: Pesquisa de campo (2010).

En relación al estilo de liderazgo adoptado en las organizaciones analizadas (Cuadro 11), en promedio, los encuestados están en desacuerdo o se muestran neutrales con la afirmación de que "existe una implicación total de los empleados en la definición de los objetivos y en la preparación de las decisiones". Importa destacar la afirmación de que "el líder acepta críticas constructivas y sugerencias de mejora", con una media de 3,63 y una desviación típica de 0,842. También obtuvieron un valor medio relativamente alto las afirmaciones de que "el líder actúa como un facilitador del proceso de adopción, implementación y uso de técnicas de Contabilidad de Gestión y Control en la organización" y de que "el proceso de decisión se encuentra centralizado en la cima de la jerarquía, sin embargo existe alguna delegación de autoridad, fundamentalmente para actividades de carácter rutinario". 
Cuadro 11 - Estilo de liderazgo en los ayuntamientos

\begin{tabular}{|l||c||c||}
\hline \multicolumn{1}{|c|}{ Estilo de liderazgo (n=98) } & Media & Desviación típica \\
\hline \hline En la organización existe un estilo de liderazgo democrático y flexible & 3,52 & 0,815 \\
\hline \hline En la organización existe un estilo de liderazgo abierto al cambio & 3,60 & 0,870 \\
\hline \hline $\begin{array}{l}\text { El líder actúa como un facilitador del proceso de adopción, implementación y uso } \\
\text { de técnicas de Contabilidad de Gestión y Control en la organización }\end{array}$ & 3,62 & 0,871 \\
\hline \hline El líder acepta críticas constructivas y sugerencias de mejora & 3,63 & 0,842 \\
\hline \hline El líder emprende un proceso de comunicación constante & 3,49 & 0,852 \\
\hline \hline $\begin{array}{l}\text { El líder delega competencias y responsabilidades, estimulando la iniciativa de las } \\
\text { personas }\end{array}$ & 3,47 & 0,876 \\
\hline \hline $\begin{array}{l}\text { El líder motiva y moviliza sus subordinados hacia los objetivos globales de la } \\
\text { organización }\end{array}$ & 3,44 & 0,957 \\
\hline \hline El líder decide lo que hay que hacer, quién, cómo y cuándo debe hacerse & 3,46 & 0,839 \\
\hline $\begin{array}{l}\text { El proceso de decisión se encuentra centralizado en la cima de la jerarquía, aunque } \\
\text { existe alguna delegación de autoridad, fundamentalmente para actividades de } \\
\text { carácter rutinario }\end{array}$ & 3,61 & 0,820 \\
\hline \hline $\begin{array}{l}\text { El líder consulta sus subordinados antes del establecimiento de objetivos y la toma } \\
\text { de decisiones }\end{array}$ & 3,21 & 0,900 \\
\hline \hline El líder presenta el problema al grupo e intenta obtener consenso & 3,16 & 0,893 \\
\hline \hline $\begin{array}{l}\text { Existe una implicación total de los empleados en la definición de los objetivos y la } \\
\text { preparación de las decisiones }\end{array}$ & 2,96 & 0,907 \\
\hline \hline
\end{tabular}

Fuente: Pesquisa de campo (2010).

Por otro lado, el 65,3\% de los ayuntamientos poseen una estrategia organizativa, el 66,7\% y el $61,6 \%$, respectivamente, tienen una misión y visión claramente definidas, el 68,7\% tienen objetivos estratégicos claramente definidos y el 52\% realizan un proceso de planificación estratégica. Las organizaciones encuestadas consideran que la utilización de indicadores de distinta naturaleza es útil o muy útil (Cuadro 12). No obstante, los indicadores/medidas financieras son los más utilizados, con un valor medio superior y una desviación típica inferior, demostrando coincidencia de opiniones entre los encuestados. En cuanto a los indicadores orientados a medir los procesos, su utilización es considerada media (el 46,3\%). Importa subrayar que 18 ayuntamientos (el 18,9\%) señalaron una utilización alta o muy alta y 33 ayuntamientos (el 34,7\%) una utilización nula o baja.

En lo que concierne a los indicadores orientados a medir los resultados, un porcentaje significativo de encuestados (el 49,5\%) indicaron que la utilización de dichos indicadores es media. Importa subrayar que 22 ayuntamientos (el 23,1\%) señalaron una utilización alta o muy alta y 26 ayuntamientos (el 27,4\%) una utilización nula o baja. En lo que respecta a la existencia de sistemas de incentivos (recompensas o penalizaciones) vinculados a indicadores, el 87,9\% de los ayuntamientos de la muestra (87 ayuntamientos) indicaron que no poseen dichos sistemas. Además, el grado de aceptación de los empleados en relación al sistema de incentivos es considerado medio (el 66,7\%). 
Cuadro 12 - Utilidad de los indicadores/medidas en los ayuntamientos

\begin{tabular}{||l||c|c||}
\hline \multicolumn{1}{|c||}{ Indicadores/ Medidas (n=96) } & Media & Desviación típica \\
\hline \hline Financieras & 4,32 & 0,741 \\
\hline \hline No financieras & 3,81 & 0,837 \\
\hline \hline Internas & 3,89 & 0,857 \\
\hline \hline Externas & 3,77 & 0,864 \\
\hline \hline A corto plazo & 3,82 & 0,871 \\
\hline \hline A largo plazo & 3,96 & 0,893 \\
\hline \hline Cualitativas & 3,89 & 0,869 \\
\hline \hline Cuantitativas & 3,98 & 0,841 \\
\hline
\end{tabular}

Fuente: Pesquisa de campo (2010).

En relación al grado de intensidad tecnológica/automatización de la organización, el 50,5\% de los 97 ayuntamientos lo calificaron como medio. Importa señalar que 49 ayuntamientos (el $47,4 \%$ ) consideraron que es alto o muy alto y solo 2 ayuntamientos (el 2,1\%) indicaron que es bajo. En lo que respecta a la comunicación dentro de la organización es razonable o fluida, tanto a nivel vertical como a nivel horizontal. Finalmente, la casi totalidad de los ayuntamientos (el 89,9\%) siguen un proceso formalizado de realización, aprobación y seguimiento del presupuesto organizativo.

\subsubsection{Conocimiento del CMI}

En lo que respecta al conocimiento de la herramienta de gestión CMI por parte de los encuestados (Cuadro 13), hemos observado que, con carácter general, las organizaciones conocen el CMI, lo que indica que dicha herramienta está bastante divulgada en Portugal. No obstante, también es importante el porcentaje de encuestados que manifestaron desconocer (el 33,3\%) o conocer poco (el 24,2\%) el CMI.

Cuadro 13 - Conocimiento del CMI en los ayuntamientos

\begin{tabular}{|l||c||c|}
\hline \multicolumn{1}{|c||}{ Conocimiento } & Ayuntamientos & \% \\
\hline \hline No conocen & 33 & 33,3 \\
\hline \hline Conocen poco & 24 & 24,2 \\
\hline \hline Conocen & 31 & 31,3 \\
\hline \hline Conocen bien & 11 & 11,1 \\
\hline \hline \multicolumn{1}{|c|}{ Total } & $\mathbf{9 9}$ & $\mathbf{1 0 0}$ \\
\hline
\end{tabular}

Fuente: Pesquisa de campo (2010).

Los encuestados que indicaron conocer el CMI fueron preguntados sobre cómo obtuvieron el conocimiento acerca del mismo (Cuadro 14). Nuestro propósito con esta pregunta era evaluar cuáles habían sido sus principales fuentes de información. En concreto, si el CMI formaba parte del contenido programático en el ámbito de la formación de nivel superior o de cursos, seminarios, conferencias y workshops a los que hubieran asistido los encuestados, si las organizaciones obtuvieron el conocimiento sobre el CMI a través de consultores, otros gerentes o proveedores de 
software, o si se ha derivado de la lectura de libros y/o artículos en revistas. Por el análisis de las respuestas obtenidas, las principales fuentes de información señaladas han sido los libros o artículos publicados en revistas especializadas (el 50,8\%), así como las revistas no especializadas, Newsletter e Internet (el 42,9\%). No obstante, también un porcentaje significativo de encuestados revelaron que habían obtenido conocimiento del CMI a través de la participación en cursos, seminarios, conferencias y workshops (el 36,5\%) y de la formación académica (el 34,9\%).

Cuadro 14 - Fuentes de información sobre el CMI en los ayuntamientos

\begin{tabular}{||l||c|c|}
\hline \multicolumn{1}{|c|}{ Fuentes de información } & Ayuntamientos & $\%$ \\
\hline \hline Consultores & 8 & 12,7 \\
\hline \hline Proveedores de software & 2 & 3,2 \\
\hline \hline Cursos, seminarios, conferencias y workshops & 23 & 36,5 \\
\hline \hline Libros o artículos en revistas especializadas & 32 & 50,8 \\
\hline \hline Revistas no especializadas, Newsletter e Internet & 27 & 42,9 \\
\hline \hline Gestores de otras organizaciones & 6 & 4,8 \\
\hline \hline Participación en proyectos relacionados con el CMI & 22 & 9,5 \\
\hline \hline Materia enseñada durante la formación académica & & 34,9 \\
\hline
\end{tabular}

Fuente: Pesquisa de campo (2010).

Con el objetivo de identificar cuál es la interpretación de los encuestados sobre el concepto de CMI, les presentamos un conjunto de afirmaciones y les pedimos que indicaran el grado en que estaban de acuerdo con las mismas, en una escala que variaba entre el desacuerdo y el acuerdo total. La observación del Cuadro 15 revela que gran parte de los encuestados está de acuerdo o coincide totalmente con la afirmación de que "el CMI es una herramienta de gestión del cambio y de alineamiento estratégico". Este resultado es importante en la medida que se trata de la definición más reciente encontrada en los últimos libros publicados por Kaplan y Norton (2007a, 2007b, 2008), lo que revela interés y una actualización constante por parte de los encuestados respecto a esta temática. Además destaca también la consideración del CMI como "un sistema de gestión e implementación de la estrategia, que favorece una mejor articulación y comunicación de la misión y objetivos organizacionales". Por otro lado, y previsiblemente, la mayor parte de los encuestados están en desacuerdo o discrepan totalmente con la consideración del CMI como "una moda" o solo como "una colección de medidas financieras y no financieras".

En la interpretación de las respuestas podemos concluir que existe un buen entendimiento del concepto y filosofía inherentes al CMI, lo cual puede atribuirse al hecho de que se ha producido una gran divulgación de esta herramienta en los últimos años, con constante publicación de libros o artículos en revistas y la organización de cursos, seminarios, conferencias y workshops respecto a esta temática. 
Cuadro 15 - Interpretación del concepto de CMI en los ayuntamientos

\begin{tabular}{|l|c|c|}
\hline \multicolumn{1}{|c|}{ Interpretación del CMI } & Media & Desviación típica \\
\hline \hline El CMI es una moda & 2,30 & 0,843 \\
\hline \hline El CMI es una colección de medidas financieras y no financieras & 3,03 & 0,966 \\
\hline \hline El CMI es un sistema de evaluación y medición del desempeño & 3,72 & 0,799 \\
\hline \hline El CMI es un sistema de gestión e implementación de la estrategia & 4,05 & 0,561 \\
\hline \hline $\begin{array}{l}\text { El CMI completa medidas financieras del desempeño pasado con medidas } \\
\text { operativas de desempeño y crecimiento futuro }\end{array}$ & 3,97 & 0,576 \\
\hline \hline El CMI vincula la misión y estrategia organizacional a medidas objetivas & 4,02 & 0,532 \\
\hline \hline Trabajar con el CMI permite reformular la estrategia, haciéndola más concreta & 4,02 & 0,532 \\
\hline $\begin{array}{l}\text { El CMI favorece una mejor articulación y comunicación de la misión y objetivos de } \\
\text { la empresa }\end{array}$ & 4,10 & 0,473 \\
\hline \hline El CMI es una herramienta de gestión del cambio y de alineamiento estratégico & 4,15 & 0,477 \\
\hline
\end{tabular}

Fuente: Pesquisa de campo (2010).

\subsubsection{Utilización del CMI}

Con el propósito de conocer en qué medida está o no generalizada la utilización del CMI, hemos preguntado a los encuestados que conocen el CMI sobre el grado de utilización del mismo (Cuadro 16). A este respecto, los resultados obtenidos revelan que el nivel de implementación del CMI en los ayuntamientos portugueses es bastante reducido (el 4,8\%). Por otro lado, un elevado porcentaje de encuestados señalaron que no utilizaban ni pretendían utilizar el CMI (el 74,6\%). Así, en la mejor de las hipótesis, considerando que el proceso de implementación llegue a terminarse y que las expectativas de implementación futura se concreten, la tasa de implementación se situaría alrededor del 25,4\% (16 ayuntamientos). No obstante, creemos que la utilización del CMI en los ayuntamientos portugueses va aumentar en el futuro, teniendo en cuenta las exigencias de mayor responsabilidad y el reconocimiento de la necesidad de aumentar el número de medidas no financieras en los sistemas de medición del desempeño.

Cuadro 16 - Utilización del CMI en los ayuntamientos

\begin{tabular}{||l||c|c||}
\hline \multicolumn{1}{|c||}{ Utilización } & Ayuntamientos & \% \\
\hline \hline No utilizan ni pretenden utilizar & 47 & 74,6 \\
\hline \hline Utilizan actualmente & 3 & 4,8 \\
\hline \hline Esperan implementarlo en el futuro & 13 & 20,6 \\
\hline \hline Total & $\mathbf{6 3}$ & $\mathbf{1 0 0}$ \\
\hline
\end{tabular}

Fuente: Pesquisa de campo (2010).

El Cuadro 17 recoge los resultados obtenidos en lo que respecta a las principales razones para no implementar o abandonar la implementación del CMI. Así, se destaca claramente como razón el reducido conocimiento del CMI (el 75,6\%). Por otra parte, un importante porcentaje de encuestados han señalado también la falta de comprensión de los beneficios derivados de la implementación del CMI (el 40\%) y la falta de compromiso, disponibilidad y apoyo por parte de la alta dirección (el 26,7\%). Además de las presentadas en el cuestionario, los encuestados han 
apuntado como razones la falta de capacidad y autonomía para implementar el CMI y el desconocimiento de otras organizaciones con la misma naturaleza que han implementado el CMI.

Cuadro 17 - Razones para la no adopción o para el abandono del CMI en los ayuntamientos

\begin{tabular}{|c|c|c|}
\hline Razones & 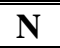 & $\%$ \\
\hline El CMI no se ajusta a la cultura y a la misión de la organización & 9 & 20 \\
\hline La organización utiliza otras herramientas similares y está satisfecha & 3 & 6,7 \\
\hline $\begin{array}{l}\text { Dificultad en la selección de las dimensiones básicas del CMI y establecimiento de } \\
\text { interrelaciones entre ellas }\end{array}$ & 4 & 8,9 \\
\hline Dificultad de definición y medición de medidas no financieras & 9 & 20 \\
\hline Falta de comprensión de los beneficios derivados de su implementación & 18 & 40 \\
\hline Exige costes de implementación/ mantenimiento demasiado grandes & 11 & 24,4 \\
\hline Exige un consumo excesivo de tiempo & 11 & 24,4 \\
\hline Incomprensión de sus impactos a nivel organizacional (ausencia de resultados) & 8 & 17,8 \\
\hline Inadecuado soporte tecnológico/ sistema de información & 7 & 15,6 \\
\hline Reducido conocimiento del CMI & 34 & 75,6 \\
\hline Cultura organizativa (actitudes negativas y aversión/resistencia al cambio y al riesgo) & 10 & 22,2 \\
\hline Falta de compromiso y motivación por parte del personal operativo & 8 & 17,8 \\
\hline Falta de compromiso, disponibilidad y apoyo por parte de la alta dirección & 12 & 26,7 \\
\hline Dificultad para descomponer los objetivos para los niveles más bajos de la organización & 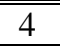 & 8,9 \\
\hline Visión a corto plazo asociada a las políticas organizativas & 11 & 24,4 \\
\hline Falta de ligación del CMI al sistema de recompensas & 2 & 4,4 \\
\hline "Cambios en la gestión/administración & 3 & 6,7 \\
\hline "Ausencia de consenso en la definición de objetivos, indicadores y metas & 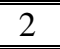 & 4,4 \\
\hline Falta de comunicación & 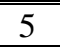 & 111,1 \\
\hline Falta de una misión/visión clara y común & 8 & 17,8 \\
\hline Otras & 2 & 4,4 \\
\hline
\end{tabular}

Fuente: Pesquisa de campo (2010).

\section{Discusión de los resultados y consideraciones finales}

En los últimos años el sector público ha estado involucrado en un contexto de cambio, con la separación de los políticos y administradores, la descentralización, la mayor cuantificación de los niveles de servicios y la medición del desempeño y un mayor énfasis en los resultados. Hoy día en las organizaciones públicas falta, la mayoría de las veces, una estructura adecuada para permitir la gestión eficaz de estas organizaciones. Los indicadores del desempeño no están vinculados a lograr la misión y los objetivos estratégicos de la Administración Pública y, por consiguiente, no son eficientes para gestionarlos. Además, el sistema de gestión debe posibilitar el alineamiento entre departamentos para retirar ventajas de las sinergias en la prestación de servicios públicos (RODRÍGUEZ et al., 2010).

La aplicación de técnicas y modelos de la gestión privada en el sector público es una consecuencia de la reforma de la Administración Pública y, por consiguiente, una cuestión de vital importancia en los días de hoy. Ciudadanos cada vez más exigentes y recursos públicos cada vez más limitados hacen con que las técnicas de gestión de empresa privada resulten no solo deseables sino imprescindibles para garantizar el correcto funcionamiento de las organizaciones públicas 
(TURULL y VIVAS, 2003). El CMI es precisamente una de esas técnicas “inmigradas" del sector privado para ser un elemento clave en la mejora de la gestión del desempeño, eficiencia y responsabilidad de muchas organizaciones del sector público. No obstante, además de perseguir los objetivos generales del sector de economía privada, el CMI en el sector público también posee características y objetivos específicos reflejados a nivel de la selección de las respectivas perspectivas y de las relaciones de causa-efecto, que exigen algunas reestructuraciones para una aplicación eficiente. Verificamos, en la revisión de la literatura, que la estructura del CMI debe modificarse en las organizaciones públicas y sin ánimo de lucro dado que, por regla general, la perspectiva financiera no asume un lugar tan relevante como lo es para la empresa privada y, de este modo, serán los clientes, entendidos en un sentido amplio, quienes pasarán a tener un papel fundamental en la estructura del CMI.

En Portugal se verifican un conjunto de problemas a nivel del funcionamiento de los servicios públicos que provocan descontentamiento en los ciudadanos, en concreto fallas de eficiencia y calidad, elevada burocracia y dificultades en el acceso a la información. Sin embargo, la realidad de las organizaciones públicas y sin fines de lucro portuguesas puede ser caracterizada por un proceso de cambio en curso, con fuerte componente legislativa, conocido por Reforma de la Administración Pública. Además, se señala la exigencia y presión cada vez mayor de los distintos stakeholders para mejorar el desempeño y la capacidad de los altos dirigentes y dirigentes intermedios para liderar la gestión del cambio y presentar resultados visibles en un corto plazo de tiempo (PINTO, 2007).

Coincidimos con Pinto (2007) de que el CMI, en particular el mapa estratégico, puede contribuir significativamente para la reforma de la Administración Pública portuguesa, en la medida que el proceso legislativo de dicha reforma envuelve el re-análisis de la misión de cada servicio público, la identificación de prioridades estratégicas y el alineamiento de recursos humanos, materiales y financieros para obtener eficiencia operativa y la mejora del desempeño. En este sentido, el CMI es indicado como una metodología capaz de alinear cambio, estrategia y performance para crear mayor valor público, siendo necesario expandir su utilización en los servicios públicos portugueses (centrales, regionales y locales), la cual deberá ser captada e interiorizada por los altos gestores y gestores intermedios. En éste proceso es fundamental identificar quienes son nuestros clientes, que tipo de servicios requieren y lo que más valoran en esa prestación de servicios, desarrollando e integrando el CMI con la estrategia global de cambio, capaz de orientar la Administración Pública portuguesa para una verdadera "cultura de la performance". Además, el citado autor comprobó, en contactos con muchos dirigentes intermedios de servicios públicos portugueses, que el CMI tiene una buena receptividad, lo que podrá facilitar su proceso de implementación, una vez que facilitará la comunicación de la estrategia en toda la organización, así 
como la definición de objetivos e indicadores balanceados en las diferentes perspectivas, según relaciones causa-efecto. En este contexto, Matos (2006) pone de manifiesto que la utilización del CMI para mejorar e incrementar la calidad de la gestión en organizaciones gubernamentales y no lucrativas no sólo es deseable como indispensable, sobre todo en Portugal donde el sector público consume más de mitad de la riqueza producida.

Según Santos (2006, p. 172), los ayuntamientos portugueses tienen que adaptar cada vez más herramientas de gestión estratégica "que les permitan crecer, prestar un mejor servicio a sus ciudadanos-munícipes, alcanzar sus objetivos, y todo esto en un contexto de muchas restricciones económicas y de recursos disponibles". Así, la necesidad de ejecutar estrategias, asociada a los nuevos desafíos enfrentados a nivel de delegación de capacidades por parte de la administración central, envuelve cambios, tanto humanos como materiales, y exige una mayor calificación de las personas y un mayor rigor en la elaboración y gestión de estrategias. En esta línea, Ferrão (2004, p. 3) señala que si los ayuntamientos portugueses pretenden mejorar su gestión deberán adoptar el CMI, en el sentido "en que es muy importante que sus responsables, a los más variados niveles, deban saber cuáles son las expectativas de sus ciudadanos-munícipes y cómo será posible satisfacerlas, dentro de todos los condicionantes existentes". En este contexto, hay que conjugar esas expectativas con las expectativas de los propios empleados y, obviamente, de sus dirigentes, en un contexto de permanente evaluación (más rigurosa y objetiva).

En lo que concierne al estudio empírico, aún reconociendo que la tasa de respuesta ha sido menor que lo deseado, creemos que es posible extraer algunas conclusiones importantes. En primer lugar, observamos que, por regla general, los ayuntamientos que constituyen la muestra se localizan en la región Norte y Centro, son de pequeña dimensión, y no están certificados en términos de sistemas de calidad y/o medioambientales.

En segundo lugar, en la caracterización general del mercado y entorno organizativo observamos que los ayuntamientos portugueses encuestados se caracterizan por poseer algunos niveles jerárquicos y por la concentración de la toma de decisiones en la alta dirección. A este respecto importa señalar que a medida que aumenta el grado de descentralización los sistemas de información de gestión deben volverse más sensitivos y sofisticados, aumentando la necesidad de controles formales en sustitución de los controles informales. Por otro lado, las organizaciones analizadas consideran que operan en un entorno en el que la incertidumbre es bastante elevada. En estos casos, se espera que haya una mayor cantidad de información procesada por las organizaciones. Con relación a este aspecto, importa subrayar que es precisamente en escenarios de incertidumbre donde el CMI se revela como más necesario, dada la necesidad de la organización en anticiparse y reaccionar a los cambios en el entorno. De la lectura de los datos, también se ha podido confirmar que la casi totalidad de las organizaciones de la muestra posee una misión, visión 
y objetivos claramente definidos y llevan a cabo un proceso de planificación estratégica. Esta información es importante y facilitará la implementación del CMI ya que son considerados, además de las perspectivas, los elementos necesarios para el diseño y construcción del mismo (KAPLAN y NORTON, 2000, 2001).

En lo que concierne a las características de la cultura organizativa hemos comprobado que las organizaciones consideran que un estilo de gestión más participativo y consultivo facilita el proceso de adopción, implementación y uso de técnicas de Contabilidad de Gestión en la organización. Con relación a estos aspectos, coincidimos con Kaplan y Norton (2007b) de que una cultura empresarial que favorezca un estilo de gestión participativo y consultivo ayuda al éxito en la implementación de nuevos sistemas de gestión en las organizaciones. En realidad, una cultura abierta supone la promoción de discusiones estratégicas, el intercambio de información y la colaboración, reduciendo la resistencia al cambio y facilitando la implementación de nuevos sistemas de gestión, como el CMI. El hecho de la comunicación dentro de la organización ser considerada por parte de los encuestados como razonable o fluida, tanto a nivel vertical como a nivel horizontal, también es un resultado importante. Entendemos, a semejanza de Fernández (2001), que una comunicación organizacional efectiva, es decir, una comunicación abierta, clara, transparente y fluida, es un elemento esencial desde el inicio del proceso de implementación del CMI, ya que puede facilitar la comprensión, interiorización y aceptación del CMI por parte de las personas que trabajan en la organización.

En tercer lugar, transcurridos veinte años desde la presentación del CMI por Kaplan y Norton, y pese haber recibido una fuerte promoción por parte de los académicos y las empresas de consultoría y de software, hemos constatado que esta herramienta, si bien es conocida por la generalidad de las organizaciones encuestadas, es aún poco utilizada en los ayuntamientos portugueses, aún cuando un alto porcentaje de las organizaciones de la muestra han desarrollado y usan indicadores de desempeño, tanto para medir los resultados como procesos. Por otro lado, hemos concluido que existe un buen entendimiento del concepto y filosofía inherentes al CMI, lo cual puede atribuirse al hecho de que se ha producido una gran divulgación de esta herramienta en los últimos años, con constante publicación de libros o artículos en revistas y la organización de cursos, seminarios, conferencias y workshops respecto a esta temática.

En definitiva, aunque sean notorias las diferencias entre los entornos privado y público, se ha comprobado que el CMI, una vez que se efectúen algunas adaptaciones, es perfectamente aplicable en el ámbito de actuación de las entidades públicas y sin fines de lucro. No obstante, la definición e implementación del CMI en dichas entidades es un proceso extremamente difícil y lento, requiriendo un fuerte apoyo e implicación de la alta dirección y una definición cuidada y clara de sus clientes. Así, creemos que el CMI puede ser una herramienta útil para tomar decisiones 
en entidades públicas y sin fines de lucro, puesto que, con unos pocos indicadores monetarios (cuantitativos) y no monetarios (cualitativos), agrupa información del conjunto de la organización y posibilita la evaluación del cumplimento de los objetivos de la misma. A este respecto, coincidimos con Cavaco y Sarrico (2007) en la consideración de que el sector público constituye un terreno propicio a la utilización del CMI, ya que permite alinear los objetivos con la estrategia, fundamental sobre todo cuando los recursos son escasos, y favorece el aprendizaje continuo, eliminando la necesidad de seleccionar un tipo de sistema de control para cada situación. Así, la única condición esencial para su aplicación es la de que la organización posea una clara definición de su estrategia y tenga un equipo motivado a invertir esfuerzos en su implementación.

\begin{abstract}
The quest for rationalization concerning the public resources' management and the need to improve the quality of the services provided, items related to the "new public management" model, implicated the application on public entities of the concepts and the practices previously developed and used in the private sector. Consequently, as a statement of that purpose, several public and nonprofit organizations have expressed specific interest in implementing the Balanced Scorecard (BSC) tools. This paper intends to provide some ideas related to the importance, the characteristics and the necessary adaptations of the BSC tools, concerning their application to public and nonprofit organizations, and investigate if the Portuguese city councils are familiar with and use the BSC tools. Accordingly, we have sent an enquiry to all the 308 Portuguese city councils, having attained a response rate of $32.5 \%$. The results suggest that, although the majority of respondents claimed to be familiar with the BSC tools, its use in these organizations is very limited.
\end{abstract}

Key-words: balanced scorecard, public sector, management accounting.

\title{
Referencias
}

AIDEMARK, L. Balanced Scorecard in Health Care: Experiences from Trials with Balanced Scorecards in Five Country Councils. In: WORKSHOP ON PERFORMANCE MEASUREMENT AND MANAGEMENT, 2001, Nice, p. $19-44$.

AIBAR, C. Diseño y Comunicación de Instrumentos de Medición del Rendimiento en el Marco de la Nueva Gestión Pública. Revista Española de Financiación y Contabilidad, v.XXXIII, n.123, p. 991-1024, 2004.

ATKINSON, A.; EPSTEIN, M. Measure for Measure: Realizing the Power of the Balanced Scorecard. CMA Management, v.74, n.7, p. 22-28, 2000.

AX, C.; BJORNENAK, T. Bundling and Diffusion of Management Accounting Innovations - The Case of the Balanced Scorecard in Sweden. Management Accounting Research, v.16, n.1, p. 1-20, 2005.

BASTIDAS, E.; MORENO, Z. El Cuadro de Mando Integral en la Gestión de las Organizaciones del Sector Público. Caso: Universidad Centroccidental Lisandro Alvarado. Revista Universo Contábil, v.2, n.3, p. 104-118, 2006.

BASTIDAS, E.; RIPOLL, V. Una Aproximación a las Implicaciones del Cuadro de Mando Integral en las Organizaciones del Sector Público. Revista Compendium, n.11, p. 23-41, 2003.

BLANCO, M.; AIBAR, B.; AIBAR, C. El Papel de los Indicadores de Gestión en la Implantación y Evaluación de la Filosofía de Calidad en la Administración Local: Estudio de un Caso. Tékhne - Revista de Estudos Politécnicos, v.5, n.8, p. 139-159, 2007. 
BLANCO, M.; BARROS, F.; CANTORNA, S.; AIBAR, B. Diseño de un Esquema de Información para la Gestión Estratégica de Entidades Camerales en el Contexto de la Globalización y el Conocimiento. In: CONGRESO AECA 11, 2001, Madrid.

BOCCI, F. A New Balanced Scorecard Architecture for the Public Sector. Perspectives on Performance, v.4, n.3, p. 30-32, 2005

BORONAT, G. Qué Ventajas Aporta el Balanced Scorecard en la Gestión Pública. Estrategia Financiera, n.211, p. 46-52, 2004.

BRIGNALL, S.; MODELL, S. An Institutional Perspective on Performance Measurement and Management in the New Sector Public. Management Accounting Research, v.11, n.3, p. 281-306, 2000.

CARMONA, S.; GRÖNLUND, A. Measures vs Actions: The BSC in Swedish Law Enforcement. International Journal of Operations \& Production Management, v.23, n.11/12, p. 1475-1496, 2003.

CARVAlHO, C.; AZEVEDO, G. Balanced Scorecard - Sistema de Informação vs Sistema de Gestão. Revista Estudos do I.S.C.A.A., n.6/7, p. 55-78, 2001.

CARVAlho, J.; FERnANDES, M.; CAMÕES, P.; JORGE, S. Anuário Financeiro dos Municípios Portugueses 2007. Lisboa: Câmara dos Técnicos Oficiais de Contas, 2009.

CAVACO, A.; SARRICO, C. Exequibilidade da Utilização do Balanced Scorecard num Hospital do Sector Público Administrativo. In: CONGRESSO NACIONAL DA ADMINISTRAÇÃO PÚBLICA 5, 2007, Instituto Nacional de Administração, Lisboa.

CHAN, Y. Performance Measurement and Adoption of the Balanced Scorecards: A Survey of Municipal Governments in the USA and Canada. The International Journal of Public Sector Management, v.17, n.2/3, p. 201-222, 2004.

DAVILA, A. Nuevas Herramientas de Control: El Cuadro de Mando Integral. IESE: Revista de Antiguos Alumnos, n.75, p. 34-42, 1999.

DEEM, J.; BARNES, B.; SEGAL, S.; PREZIOSI, R. The Relationship of Organizational Culture to Balanced Scorecard Effectiveness. Advanced Management Journal, v.75, n.4, p. 31-39, 2010.

ELLINGSON, D.; WAMBSGANSS, J. Modifying the Approach to Planning and Evaluation in Governmental Entities: A Balanced Scorecard Approach. Journal of Public Budgeting, Accounting \& Financial Management, v.13, n.1, p. 103-120, 2001.

FÄLTHOLM, Y.; NILSSON, K. Business Process Re-Engineering and Balanced Scorecard in Swedish Public Sector Organizations: Solutions for Problems or Problems for Solutions? International Journal of Public Administration, v.33, n.6, p. 302-310, 2010.

FERNÁNDEZ, A. El Balanced Scorecard: Ayudando a Implantar la Estrategia. Revista de Antiguos Alumnos, n.81, p. 31-42, 2001.

FERRÃO, F. O Balanced Scorecard nas Autarquias. Jornal Expresso, Edição de 25 de Setembro, p. 4-6, 2004.

GOMES, A.; CARVALHO, J.; RIBEIRO, N.; NOGUEIRA, S. O Balanced Scorecard Aplicado à Administração Pública. Lisboa: Publisher Team, 2007.

HOOD, C. The New Public Management in the 1980s: Variations on a Theme. Accounting, Organizations and Society, v.20, n.2/3, p. 93-109, 1995.

HOOD, C. A Public Management for all Seasons? Public Administration, v.69, n.1, p. 3-19, 1991.

HOQUE, Z. Securing Institutional Legitimacy or Organizational Effectiveness? A Case Examining the Impact of Public Sector Reform Initiatives in an Australian Local Authority. The International Journal of Public Sector Management, v.18, n.4/5, p. 367-382, 2005.

HOQUE, Z.; ADAMS, C. The Rise and Use of Balanced Scorecard Measures in Australian Government Departments. Financial Accountability \& Management, v.27, n.3, p. 308-334, 2011. 
HORVÁTH \& PARTNERS. Dominar el Cuadro de Mando Integral: Manual Práctico Basado en Más de 100 Experiencias. Barcelona: Ediciones Gestión 2000, 2003.

JOHNSEN, A. Balanced Scorecard: Theoretical Perspectives and Public Management Implications. Managerial Auditing Journal, v.16, n.6, p. 319-330, 2001.

cross ref

KAPLAN, R. Strategic Performance Measurement and Management in Nonprofit Organizations. Nonprofit Management \& Leadership, v.11, n.3, p. 353-370, 2001.

cross ref

KAPLAN, R.; NORTON, D. Mastering the Management System. Harvard Business Review, v.86, n.1, p. 62-77, 2008.

KAPLAN, R.; NORTON, D. Using the Balanced Scorecard as a Strategic Management System. Harvard Business Review, v.85, n.7/8, p. 150-161, 2007a.

KAPLAN, R.; NORTON, D. Alignment: Cómo Alinear la Organización a la Estrategia a través del Balanced Scorecard. Barcelona: Ediciones Gestión2000, 2007b.

KAPLAN, R.; NORTON, D. Mapas Estratégicos: Convirtiendo los Activos Intangibles en Resultados Tangibles. Barcelona: Ediciones Gestión2000, 2004.

KAPLAN, R.; NORTON, D. The Strategy Focused Organization: How Balanced Scorecard Companies Thrive in the New Business Environment. Massachusetts: Harvard Business School Press, 2001.

KAPLAN, R.; NORTON, D. El Cuadro de Mando Integral (The Balanced Scorecard). Barcelona: Ediciones Gestión2000, 2000.

KAPLAN, R.; NORTON, D. Using the Balanced Scorecard as a Strategic Management System. Harvard Business Review, v.74, n.1, p. 75-85, 1996a.

KAPLAN, R.; NORTON, D. Linking the Balanced Scorecard to Strategy. California Management Review, v.39, n.1, p. 53-79, $1996 \mathrm{~b}$.

KAPLAN, R.; NORTON, D. The Balanced Scorecard - Measures that Drive Performance. Harvard Business Review, v.70, n.1, p. 71-79, 1992.

KLOOT, L.; MARTIN, J. Strategic Performance Management: A Balanced Approach to Performance Management Issues in Local Government. Management Accounting Research, v.11, n.2, p. 231-251, 2000.

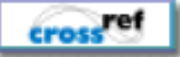

LANG, S. Balanced Scorecard and Government Entities. The CPA Journal, v.74, n.6, p. 48-51, 2004.

LAPSLEY, I. Accounting and the New Public Management: Instruments of Substantive Efficiency or a Rationalising Modernity? Financial Accountability \& Management, v.15, n.3/4, p. 201-207, 1999.

cross ref

LAWSON, R.; STRATTON, W.; HATCH, T. Scorecarding in the Public Sector: Fad or Tool of Choice? Government Finance Review, v.23, n.3, p. 48-52, 2007.

MARIN, J. The Impact of Strategic Planning and the Balanced Scorecard Methodology on Middle Managers' Performance in the Public Sector. International Journal of Business and Social Science, v.3, n.1, p. 114-127, 2012.

MATOS, L. A Utilização do Balanced Scorecard para Monitorear o Desempenho de um Hospital. 2006. Dissertação (Mestrado em Sistemas de Informação), Universidade do Minho, Braga, Portugal.

MICHELI, P.; KENNERLEY, M. Performance Measurement Frameworks in Public and Non-Profit Sectors. Production Planning \& Control, v.16, n.2. p. 125-134, 2005.

cross ref 
MODELL, S. Bundling Management Control Innovations: A Field Study of Organisational Experimenting with Total Quality Management and the Balanced Scorecard. Accounting, Auditing and Accountability Journal, v.22, n.1, p. 59-90, 2009.

cross ref

MODELL, S. Performance Measurement Myths in the Public Sector: A Research Note. Financial Accountability \& Management, v.20, n.1, p. 39-55, 2004.

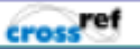

MORA, A.; VIVAS, C. Nuevas Herramientas de Gestión Pública: El Cuadro de Mando Integral. Madrid: AECA Monografías, 2001.

NILSSON, K. Expectations from Administrative Reforms: An Attempt to Implement the Balanced Scorecard in a Public Sector Organization. International Journal of Public Administration, v.33, n.14, p. 822-, 2010.

NIVEN, P. El Cuadro de Mando Integral Paso a Paso. Barcelona: Ediciones Gestión2000, 2003.

OLVE, N.; ROY, J.; WETTER, M. Implantando y Gestionando El Cuadro de Mando Integral (Performance Drivers). Barcelona: Ediciones Gestión2000, 2002.

OLVE, N.; ROY, J.; WETTER, M. Performance Drivers: A Practical Guide to Using the Balanced Scorecard. New York: John Wiley \& Sons, 1999.

PALLOT, J. Beyond NPM: Developing Strategic Capacity. Financial Accountability \& Management. v.15, n.3/4, p. 419-426, 1999.

cross ref

PEDRO, J. O Balanced Scorecard (BSC) no Sector Público. Informação \& Informática, n.28, p. 14-23, 2004.

PEDRO, J. Performance no Sector Público. Revista TOC, n.43, p. 60-63, 2003.

PINTO, F. Balanced Scorecard - Alinhar Mudança, Estratégia e Performance nos Serviços Públicos. Lisboa: Edições Sílabo, 2007.

POLliTT, C.; SUMMA, H. Trajectories of Reform: Public Management Change in Four Countries. Public Money and Management, v.17, n.1, p. 7-18, 1997.

cross ref

RAUTIAINEN, A. The Interrelations of Decision-Making Rationales around BSC Adoptions in Finnish Municipalities. International Journal of Productivity and Performance Management, v.58, n.8, p. 787-802, 2009.

cross ref

RODRÍGUEZ, M.; LÓPEZ, A.; ORTIZ, D. Implementing the Balanced Scorecard in Public Sector Agencies: An Experience in Municipal Sport Services. Academia, n.45, p. 116-139, 2010.

RODRÍGUEZ, M.; ORTIZ, D. El Cuadro de Mando Integral y su Aplicación al Control de la Gestión en las Administraciones Públicas. Harvard Deusto, Finanzas \& Contabilidad, n.50, p. 22-32, 2002.

RUAS, E. Criação de Indicadores Estratégicos para o Instituto de Pesquisas Tecnológicas do Estado de São Paulo. RAE-eletrônica, v.2, n.1, p. 1-18, 2003.

SANTOS, R. Balanced Scorecard em Portugal: Visão, Estratégia e Entusiasmo. Lisboa: Gestão Plus Edições, 2006.

SANTOS, M.; FIDALGO, E. Un Análisis de la Flexibilidad del Cuadro de Mando Integral (CMI) en su Adaptación a la Naturaleza de las Organizaciones. Revista Iberoamericana de Contabilidad de Gestión, v.II, n.4, p. 85-116, 2004.

SANTOS, M.; FIDALGO, E. Del Cuadro de Mando al Cuadro de Mando Integral: Las "Perspectivas" en la Gestión Sanitaria. In: JORNADA ASEPUC 8, 2003, Universidad de Cantabria, p. 239-252. 
SARTORIUS, K.; TROLLIP, N.; EITZEN, C. Performance Measurement Frameworks in a State Controlled Research Organization: Can the Balanced Scorecard (BSC) Be Modified? South African Journal of Business Management, v.41, n.2, p. 51-64, 2010.

SHARMA, B.; GADENNE, D. Balanced Scorecard Implementation in a Local Government Authority: Issues and Challenges. Australian Journal of Public Administration, v.70, n.2, p. 167-184, 2011.

cross ref

SILVA FILHO, J.; RODRÍGUEZ, R. Una Nueva Visión del Cuadro de Mando Integral para el Sector Público. Revista Iberoamericana de Contabilidad de Gestión, v.II, n.4, p. 117-148, 2004.

TER BOGT, H. Management Accounting Change and New Public Management in Local Government: A Reassessment of Ambitions and Results - An Institutionalist Approach to Accounting Change in the Dutch Public Sector. Financial Accountability \& Management, v.24, n.3, p. 209-241, 2008.

cross ref

TER BOGT, H.; VAN HELDEN, G. Accounting Change in Dutch Government: Exploring the Gap between Expectations and Realizations. Management Accounting Research, v.11, n.2, p. 263-279, 2000.

cross ref

TURULL, J.; VIVAS, C. El Cuadro de mando Integral en la Administración Pública: El Caso del Ayuntamiento de Sant Cugat del Vallès. In: CONGRESO INTERNACIONAL DEL CLAD 8, 2003, Panamá.

VOELKER, D.; RAHICH, J.; FRENCH, G. The Balanced Scorecard in Healthcare Organizations: a Performance Measurement and Strategic Planning Methodology. Hospital Topics, v.79, n.3, p. 13-24, 2001.

cross ref

WALSH, P. Counting for Local Experience. Australian CPA, v.70, n.10, p. 44-47, 2000.

WILSON, C.; HAGARTY, D.; GAUTHIER, J. Results Using the Balanced Scorecard in the Public Sector. Journal of Corporate Real State, v.6, n.3, p. 53-63, 2003.

cross ref

WISNIEWSKI, M.; ÓLAFSSON, S. Developing Balanced Scorecard in Local Authorities: A Comparison of Experience. International Journal of Productivity and Performance Management, v.53, n.7, p. 602-610, 2004.

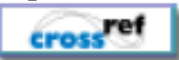

WOODS, M.; GRUBNIC, S. Linking Comprehensive Performance Assessment to the Balanced Scorecard: Evidence from Hertfordshire County Council. Financial Accountability \& Management, v.24, n.3, p. 343-361, 2008.

cross ref

YETANO, A. El Cuadro de Mando Integral en la Administración Local. Auditoría Pública, n.36, p. 39-46, 2005.

\section{Dados dos autores:}

Nome completo: Patrícia Rodrigues Quesado

Filiação institucional: Instituto Politécnico do Cávado e do Ave

Departamento: Escola Superior de Gestão

Função ou cargo ocupado: Professora Adjunta

Endereço completo para correspondência: 4750-810 Barcelos, Portugal 
Telefones para contato: 00351253802500

e-mail:pquesado@ipca.pt

\section{Nome completo: Beatriz Aibar Guzmán}

Filiação institucional: Universidad de Santiago de Compostela

Departamento: Facultad de Ciencias Económicas y Empresariales

Função ou cargo ocupado: Profesora Titular

Endereço completo para correspondência: Avda Burgo das Nacións, s/n, 15782 Santiago de

Compostela, España

Telefones para contato: 0034981563100

e-mail:beatriz.aibar@usc.es

Nome completo: Lúcia Maria Portela de Lima Rodrigues

Filiação institucional: Universidade do Minho

Departamento: Escola de Economia e Gestão

Função ou cargo ocupado: Professora Associada (com agregação)

Endereço completo para correspondência: Gualtar, 4710-057, Braga, Portugal

Telefones para contato: 00351253604559

e-mail: lrodrigues@eeg.uminho.pt

Enviado em: 09/05/2012

Aprovado em: 12/06/2012 